\title{
Synthesis, Hypoglycemic Effect, Antimicrobial and Molecular Docking Studies of Organotin(IV) Complexes Derived from $N$-Phthalimido $\beta$-Amino Acid Derivatives
}

\author{
Muhammad M. Ahmed, ${ }^{\circledR *, a}$ Nagina N. Riaz, ${ }^{a, b}$ Muhammad Kashif, ${ }^{\circledR c}$ \\ Muhammad Ashfaq, ${ }^{d}$ Muhammad N. Arshad ${ }^{a}$ and Muhammad Sajid ${ }^{a}$ \\ ${ }^{a}$ Institute of Chemical Sciences, Bahauddin Zakariya University, 60800 Multan, Pakistan \\ ${ }^{b}$ Department of Chemistry, Division of Science \& Technology, University of Education, 54770 Lahore, Pakistan \\ ${ }^{c}$ Govt. Emerson College, Bahauddin Zakariya University, 60700 Multan, Pakistan, \\ ${ }^{d}$ Department of Chemistry, The Islamia University of Bahawalpur, 63100 Bahawalpur, Pakistan
}

\begin{abstract}
$N$-Phthalimido $\beta$-amino acid derivatives, 3-phthalimido-3(2-hydroxyphenyl) propanoic acid (P2HPA) and 3-phthalimido-3(2-nitrophenyl) propanoic acid (P2NPA) with new series of diand triorganotin(IV) complexes (1-12) have been designed and synthesized. All the ligands and organotin(IV) complexes were characterized by elemental analysis, Fourier transform infrared spectroscopy (FTIR), nuclear magnetic resonance $\left({ }^{1} \mathrm{H},{ }^{13} \mathrm{C},{ }^{119} \mathrm{Sn}\right)$ spectroscopy and electron ionization mass spectrometry (EI-MS). Synthesized ligands and complexes were screened to determine the antibacterial activity and results showed that the triorganotin(IV) complexes have better activity compared to diorganotin(IV) complexes and ligands. In addition, molecular docking analysis of ligands on the catalytic pocket of sortase A (PDB ID 1T2W) showed that the ligands can bind the active amino acid residues in the pocket. The antioxidant activity was also performed by the DPPH (1,1-diphenyl-2-picrylhydrazyl radical) method and complexes showed better results than ligands. The compounds were also tested in vivo to determine the hypoglycemic activities on different groups of alloxan induced diabetic rabbits. The complexes (1-6) were found better hypoglycemic agents as they stabilized the glucose level to about $175-105 \mathrm{mg} \mathrm{dL}^{-1}$ as compared to ligand P2HPA.
\end{abstract}

Keywords: $\beta$-amino acids, organotin(IV) complexes, molecular docking, antibacterial activity, antioxidant activity, hypoglycemic effect

\section{Introduction}

Organotin(IV) compounds have gained considerable attention due to their remarkable industrial, medicinal and agricultural applications. ${ }^{1-3}$ Since the last decade, the coordination of biologically active ligands such as amino acids to tin(IV) metal ion has been strained due to its wide utilization in different areas such as antitumour, ${ }^{4}$ antiviral, ${ }^{5}$ bactericides, fungicides, ${ }^{6}$ marine antifouling paints, surface disinfectants, wood preservatives ${ }^{7}$ and various others. Several fascinating structural possibilities of amino acids and their $\beta$-analogues have been observed during their coordination to organotin(IV) groups, since they are known as potentially polydentate ligands and the organotin(IV) compounds acquire higher coordination numbers under favorable conditions. ${ }^{8}$

*e-mail: mahboobahmed@bzu.edu.pk
On the other hand, $\beta$-amino acid derivatives are considered as fundamental building blocks for various chemical precursors and these are used in pharmaceutical and agrochemical industries. ${ }^{9} 10$ Studies ${ }^{11}$ revealed that $\beta$-amino acid derivatives are also important constituents of some toxins and specialized pore-forming lipopeptides which have particular antibacterial and antifungal activity. $\beta$-Amino acid derivatives have attained the attraction in drug development because of the uniqueness of their structure, molecular recognition, biomolecular structural and functional studies. ${ }^{11-13}$

The commonness of species prone to various antimicrobial agents has amplified, resulting in increased illness, death and total cure prices, so infectious diseases are one of the foremost threats to human life. ${ }^{14,15}$ The prompt growth of multidrug-resistant pathogens is due to the overuse of antibiotics. ${ }^{16}$ The persistent increase in the extent of multidrug resistance to the existing antibiotics is 
a prime challenge to the researchers and associated with the discovery of new antimicrobial agents. ${ }^{17}$

Antioxidant moieties are microingredients that constrain lipid oxidation by preventing the commencement or proliferation of oxidizing chain reactions and are also elaborated in scavenging of free radical. ${ }^{18}$ Free radicals cause oxidative damage to lipids, proteins and deoxyribonucleic acid (DNA), eventually leading to many chronic diseases such as cancer, diabetes, aging and other degenerative diseases in humans. ${ }^{19}$

High blood glucose level was observed in a person suffering from diabetes mellitus (type-2) caused either by inappropriate production of insulin by the pancreas or by the inadequate response of body's cells towards insulin. ${ }^{20}$ Type- 2 diabetes contributes to about $90 \%$ of diabetes cases and around 150-220 million people globally suffer from diabetes every year. ${ }^{21}$ Resistant bacterial infection in diabetic patient is common. The diabetic patients have more exposure to antibiotics and have great problems with healing of infections because of reduced blood supply, which affects the body to resist against infection. Studies ${ }^{22}$ revealed that, among the bacteria isolated from wounds of diabetic patients, the number of Gram-negative was higher when compared to Gram-positive ones. Escherichia coli among Gram-negative and Staphylococcus aureus among Gram-positive were found as most common pathogens in wounds of diabetic patients. ${ }^{22}$ This very alarming situation justifies the research on novel drugs having potential against the bacterial and diabetic diseases. However, less attention has been paid to the study of antibacterial, antioxidant and antidiabetic properties of $\beta$-amino acid carboxylates and their organotin(IV) complexes, even though this class of compounds possess potential biological properties. ${ }^{23}$

Keeping in mind the above challenges, $N$-phthalimido derivatives 3-phthalimido-3(2-hydroxyphenyl) propanoic acid (P2HPA) and 3-phthalimido-3(2-nitrophenyl) propanoic acid (P2NPA) and their organotin(IV) derivatives are being reported here. Comprehensive spectroscopic analysis of the proposed ligands and corresponding complexes was performed to confirm their synthesis. All the ligands and complexes were also evaluated for in vitro antibacterial, antioxidant and in vivo antidiabetic activities to determine their potential and possible applications. Molecular docking studies using sortase A from S. aureus were also performed to better understand the mechanism of action of proposed ligands against the bacterial strain. This enzyme has vital importance due to its presence at the surface of bacteria, and it plays a fundamental role in microbial physiology. It is a frequently virulence factor which also enables the microbes to effectively interact with the environment. ${ }^{24}$

\section{Experimental}

\section{Materials and instruments}

2-Hydroxy benzaldehyde, 2-nitro benzaldehyde, malonic acid, ammonium acetate, phthalic anhydride, dibutyltin(IV) oxide, dibutyltin(IV) dichloride, tributyltin(IV) chloride, triphenyltin(IV) chloride and tricyclohexyltin(IV) chloride (Merck Chemicals, Germany) were acquired and used without further purification. All organic solvents were dried and purified as per given procedures. ${ }^{25}$ Fisher-Johns (USA) melting point apparatus was used to determine the melting points. Elemental analysis was carried out by an Eager 300 mass analyzer (USA). A Bruker FTIR (USA) spectrophotometer using OPUS software TENSOR 27 (ZnSe) covering 4000-400 $\mathrm{cm}^{-1}$ recorded the Fourier transform infrared spectroscopy (FTIR) spectra of the samples. A Bruker AM 400 NMR (USA) spectrometer recorded the ${ }^{1} \mathrm{H},{ }^{13} \mathrm{C}$ and ${ }^{119} \mathrm{Sn}$ nuclear magnetic resonance (NMR) spectra of all the compounds. The chemical shifts were reported relative to $\left(\mathrm{CH}_{3}\right)_{4} \mathrm{Si}$ and $\left(\mathrm{CH}_{3}\right)_{4} \mathrm{Sn}$ signals, which were used as internal standards. Electron ionization mass spectrometry (EI-MS) spectra were recorded on a Finnigan MAT 312 spectrometer (USA).

\section{Synthesis of $\beta$-amino acid derivatives}

The first step of the synthetic pathway followed Radionov Johnson method ${ }^{26,27}$ in which the malonic acid $(1.04 \mathrm{~g}, 10 \mathrm{mmol})$ and ammonium acetate $(0.778 \mathrm{~g}$, $10 \mathrm{mmol}$ ) with an equimolar ratio of the corresponding aldehyde were refluxed in 1-butanol. In the second step the stoichiometric amounts of the synthesized $\beta$-amino acids and phthalic anhydride were added in acetic acid and refluxed for 8 h. ${ }^{28,29}$ The mixture was allowed to cool and stand at room temperature to obtain $N$-phthalimido $\beta$-amino acid derivatives. The crystals obtained were filtered, washed and collected but at the moment crystals were not submitted to X-ray diffraction studies (Scheme 1).

3-Phthalimido-3(2-hydroxyphenyl) propanoic acid (P2HPA)

Yield $60 \%$; mp $183^{\circ} \mathrm{C}$; solubility: methanol, ethanol, acetone, $N, N$-dimethylformamide (DMF), dimethyl sulfoxide (DMSO); anal. calcd. (\%): C 45.4, H 5.8, N 8.9, found: C 45.2, H 5.7, N 8.8; FTIR $v_{\max } / \mathrm{cm}^{-1} 3000-2500$ $v(\mathrm{O}-\mathrm{H}), 1750 v(\mathrm{COO})_{\text {asym }}, 1620 v(\mathrm{COO})_{\text {sym }}, \Delta v 130,1790$ $v(\mathrm{~N}-\mathrm{CO})_{\text {asym }}, 1760 v(\mathrm{~N}-\mathrm{CO})_{\text {sym }}, 1205 v(\mathrm{C}-\mathrm{N}) ;{ }^{1} \mathrm{H}$ NMR (400 MHz, DMSO) $\delta 10.56(1 \mathrm{H}, \mathrm{s}, \mathrm{OH}), 4.90(1 \mathrm{H}, \mathrm{s}$, $\mathrm{OH}), 1.25(2 \mathrm{H}, \mathrm{d}, J 7.5 \mathrm{~Hz}, \mathrm{H} 2), 1.43(1 \mathrm{H}, \mathrm{t}, J 7.3 \mathrm{~Hz}$, H3), $7.24(1 \mathrm{H}, \mathrm{d}, J 7.1 \mathrm{~Hz}, \mathrm{H} 6), 7.41(1 \mathrm{H}, \mathrm{d}, J 7.0 \mathrm{~Hz}, \mathrm{H} 7)$, $7.70(1 \mathrm{H}, \mathrm{d}, J 7.4 \mathrm{~Hz}, \mathrm{H} 10), 7.86(1 \mathrm{H}, \mathrm{d}, J 7.8 \mathrm{~Hz}, \mathrm{H} 11)$; 


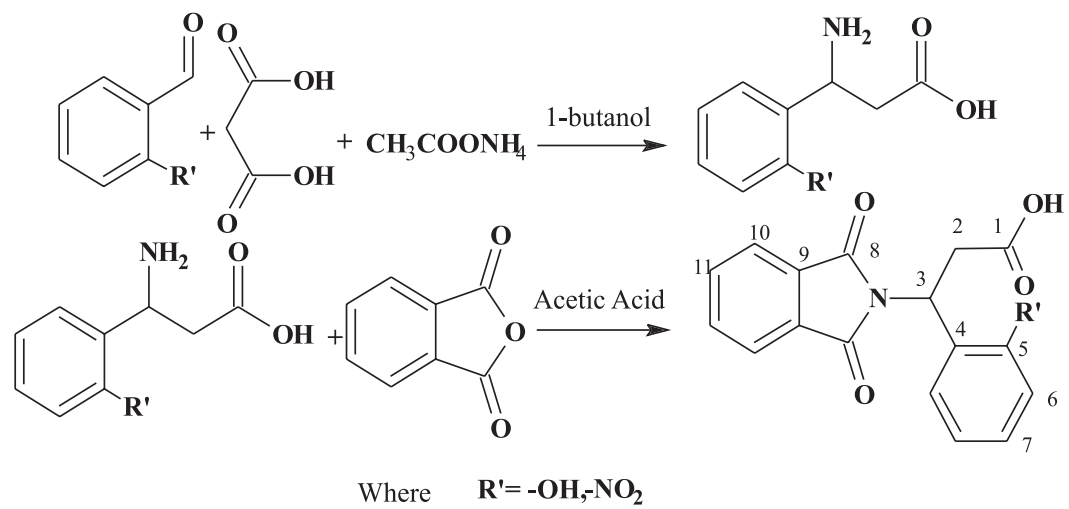

Scheme 1. Synthesis of $N$-phthalimido $\beta$-amino acid derivatives.

${ }^{13} \mathrm{C}$ NMR (100 MHz, DMSO) $\delta 169.38(\mathrm{C} 1), 45.69(\mathrm{C} 2)$, 30.09 (C3), 116.50 (C4), 118.36 (C5), 118.70 (C6), 125.20 (C7), 162.84 (C8), 130.59 (C9), 134.66 (C10), 139.16 (C11); EI-MS m/z, [C $\left.\mathrm{C}_{17} \mathrm{H}_{13} \mathrm{NO}_{5}\right]^{+}: 311(69 \%),\left[\mathrm{C}_{17} \mathrm{H}_{12} \mathrm{NO}_{4}\right]^{+}$: $294(54 \%),\left[\mathrm{C}_{16} \mathrm{H}_{12} \mathrm{NO}_{3}\right]^{+}: 266(58 \%),\left[\mathrm{C}_{16} \mathrm{H}_{11} \mathrm{NO}_{2}\right]^{+}: 249$ (100\%), [ $\left.\mathrm{C}_{10} \mathrm{H}_{7} \mathrm{NO}_{2}\right]^{+}: 173(61 \%),\left[\mathrm{C}_{8} \mathrm{H}_{4} \mathrm{NO}_{2}\right]^{+}: 146(46 \%)$, $\left[\mathrm{C}_{7} \mathrm{H}_{4} \mathrm{O}\right]^{+}: 104(68 \%),\left[\mathrm{C}_{6} \mathrm{H}_{4}\right]^{+}: 76(54 \%)$.

\section{3-Phthalimido-3(2-nitrophenyl) propanoic acid (P2NPA)}

Yield 68\%; mp $230{ }^{\circ} \mathrm{C}$; solubility: methanol, ethanol, acetone, DMF, DMSO; anal. calcd. (\%): C 49.7, H 5.8, N 8.6, found: C 49.6, H 5.9, N 8.8; FTIR $v_{\max } / \mathrm{cm}^{-1} 3300-2600$ $v(\mathrm{O}-\mathrm{H}), 1740 v(\mathrm{COO})_{\text {asym }}, 1610 v(\mathrm{COO})_{\text {sym }}, \Delta v 130,1780$ $v(\mathrm{~N}-\mathrm{CO})_{\text {asym }}, 1760 v(\mathrm{~N}-\mathrm{CO})_{\text {sym }}, 1270 v(\mathrm{C}-\mathrm{N}) ;{ }^{1} \mathrm{H}$ NMR (400 MHz, DMSO) $\delta 10.82(1 \mathrm{H}, \mathrm{s}, \mathrm{OH}), 1.29(2 \mathrm{H}, \mathrm{d}$, $J 7.3 \mathrm{~Hz}, \mathrm{H} 2), 1.50(1 \mathrm{H}, \mathrm{t}, J 7.5 \mathrm{~Hz}, \mathrm{H} 3) 7.44(1 \mathrm{H}, \mathrm{d}$, $J 7.3 \mathrm{~Hz}, \mathrm{H} 6), 7.49$ (1H, d, J 7.1 Hz, H7), 7.61 (1H, d, $J 7.5 \mathrm{~Hz}, \mathrm{H} 10), 7.96(1 \mathrm{H}, \mathrm{d}, J 7.1 \mathrm{~Hz}, \mathrm{H} 11) ;{ }^{13} \mathrm{C} \mathrm{NMR}$ (100 MHz, DMSO) $\delta 167.83$ (C1), 49.27 (C2), 36.75 (C3), 128.98 (C4), 129.73 (C5), 131.20 (C6), 133.27 (C7), 165.33(C8), 135.16 (C9), 138.20 (C10), 139.97 (C11); EI-MS $m / z,\left[\mathrm{C}_{17} \mathrm{H}_{12} \mathrm{~N}_{2} \mathrm{O}_{6}\right]^{+}: 340(45 \%),\left[\mathrm{C}_{16} \mathrm{H}_{11} \mathrm{~N}_{2} \mathrm{O}_{4}\right]^{+}$: $295(100 \%),\left[\mathrm{C}_{16} \mathrm{H}_{11} \mathrm{NO}_{2}\right]^{+}: 249(53 \%),\left[\mathrm{C}_{10} \mathrm{H}_{7} \mathrm{NO}_{2}\right]^{+}: 173$ (52\%), $\left[\mathrm{C}_{8} \mathrm{H}_{4} \mathrm{NO}_{2}\right]^{+}: 146(64 \%),\left[\mathrm{C}_{7} \mathrm{H}_{4} \mathrm{O}\right]^{+}: 104$ (59\%), $\left[\mathrm{C}_{6} \mathrm{H}_{4}\right]^{+}: 76(67 \%)$.

\section{Synthesis of organotin(IV) complexes}

Dibutyltin(IV) oxide and the corresponding $\beta$-amino acid derivative in 2:1 (monomer) and 1:1 (dimer) molar ratios have been used to synthesize diorganotin(IV) complexes $(\mathbf{1}, \mathbf{2}, \mathbf{7}, \mathbf{8})$ in ethanol and toluene $(3: 1, \mathrm{v} / \mathrm{v})$ by using Dean Stark apparatus with the removal of water azeotropically. Similarly, the silver salts of the corresponding $\beta$-amino acid derivatives and the organotin(IV) chlorides in appropriate molar ratios $(2: 1 / 1: 1)$ were refluxed for $6 \mathrm{~h}$ in toluene/ ethanol $(3: 1, \mathrm{v} / \mathrm{v})$ to synthesize compounds $(\mathbf{3 - 6}, \mathbf{9 - 1 2})$. The solvent was evaporated under vacuum. Recrystallization was made in different solvents. The procedures for the synthesis of organotin(IV) complexes were also cited in the literature $\mathrm{e}^{30-33}$ and given in Scheme 2.

Dibutyltin(IV)-di-3-phthalimido-3(2-hydroxyphenyl) propanoate (1) (monomer)

Yield $78 \%$; $\mathrm{mp} 202^{\circ} \mathrm{C}$; solubility: chloroform, acetone, DMF, DMSO, tetrahydrofuran (THF); anal. calcd. (\%): C 41.2, H 6.2, N 5.8, found: C 41.3, H 6.2, N 5.10; FTIR $v_{\max } / \mathrm{cm}^{-1} 1630 v(\mathrm{COO})_{\text {asym }}, 1470 v(\mathrm{COO})_{\text {sym }}, \Delta v 160,1728$ $v(\mathrm{~N}-\mathrm{CO})_{\text {asym }}, 1690 v(\mathrm{~N}-\mathrm{CO})_{\text {sym }}, v(\mathrm{C}-\mathrm{N}) 1020,519 v(\mathrm{Sn}-\mathrm{C})$, $505 v(\mathrm{Sn}-\mathrm{O}) ;{ }^{1} \mathrm{H}$ NMR (400 MHz, DMSO) $\delta 4.58(1 \mathrm{H}$, s, OH), 1.29 (2H, d, J 7.5 Hz, H2), $1.50(1 \mathrm{H}, \mathrm{t}, J 7.3 \mathrm{~Hz}$, H3), 6.29 (1H, d, J 7.1 Hz, H6), 6.97 (1H, d, J 7.0 Hz, H7), 7.49 (1H, d, J 7.4 Hz, H10), 7.72 (1H, d, J 7.8 Hz, H11), $1.15(2 \mathrm{H}, \mathrm{t}, J 7.0 \mathrm{~Hz}, \mathrm{Ha}), 1.79(2 \mathrm{H}, \mathrm{m}, \mathrm{Hb}), 2.01(2 \mathrm{H}$, m, Hc), 0.85 (3H, t, J 7.0 Hz, Hd); ${ }^{13} \mathrm{C}$ NMR (100 MHz, DMSO) $\delta 170.25$ (C1), 45.15 (C2), 33.40 (C3), 116.33 (C4), 125.58 (C5), 129.49 (C6), 130.47 (C7), 163.85 (C8), 144.75 (C9), 139.73 (C10), 132.53 (C11), 27.13 (Ca), $26.48(\mathrm{Cb}), 23.50(\mathrm{Cc}), 13.15(\mathrm{Cd}) ;{ }^{119} \mathrm{Sn} \mathrm{NMR}\left(\mathrm{CH}_{3}\right)_{4} \mathrm{Sn}$ : 190.35; EI-MS $m / z,\left[\left(\mathrm{C}_{4} \mathrm{H}_{9}\right)_{2} \mathrm{Sn}\left\{\mathrm{O}_{2} \mathrm{CC}_{16} \mathrm{H}_{12} \mathrm{NO}_{3}\right\}_{2}\right]^{+}$: $850(20 \%), \quad\left[\left(\mathrm{C}_{4} \mathrm{H}_{9}\right)_{2} \mathrm{SnO}_{2} \mathrm{C}\left\{\mathrm{C}_{16} \mathrm{H}_{12} \mathrm{NO}_{3}\right\}_{2}\right]^{+}$: $806(34 \%), \quad\left[\left(\mathrm{C}_{4} \mathrm{H}_{9}\right) \mathrm{Sn}\left\{\mathrm{O}_{2} \mathrm{CC}_{16} \mathrm{H}_{12} \mathrm{NO}_{3}\right\}_{2}\right]^{+}$: $793(46 \%), \quad\left[\left(\mathrm{C}_{4} \mathrm{H}_{9}\right) \mathrm{SnO}_{2} \mathrm{C}\left\{\mathrm{C}_{16} \mathrm{H}_{12} \mathrm{NO}_{3}\right\}_{2}\right]^{+}$: $749(37 \%), \quad\left[\left(\mathrm{C}_{4} \mathrm{H}_{9}\right) \mathrm{Sn}\left\{\mathrm{C}_{16} \mathrm{H}_{12} \mathrm{NO}_{3}\right\}_{2}\right]^{+}: 705$ (51\%), $\left[\left(\mathrm{C}_{4} \mathrm{H}_{9}\right)_{2} \mathrm{Sn}\left\{\mathrm{O}_{2} \mathrm{CC}_{16} \mathrm{H}_{12} \mathrm{NO}_{3}\right\}\right]^{+}: 541$ (30\%), $\left[\left(\mathrm{C}_{4} \mathrm{H}_{9}\right)_{2} \mathrm{Sn}\left\{\mathrm{C}_{16} \mathrm{H}_{12} \mathrm{NO}_{3}\right\}\right]^{+}: 497(100 \%),\left[\left(\mathrm{C}_{4} \mathrm{H}_{9}\right)\right.$ $\left.\operatorname{Sn}\left\{\mathrm{C}_{16} \mathrm{H}_{12} \mathrm{NO}_{3}\right\}\right]^{+}: 440(27 \%),\left[\operatorname{Sn}\left\{\mathrm{C}_{16} \mathrm{H}_{12} \mathrm{NO}_{3}\right\}\right]^{+}: 383$ (42\%), $\left[\mathrm{C}_{16} \mathrm{H}_{12} \mathrm{NO}_{3}\right]^{+}: 265$ (39\%), [Sn] : 119 (52\%), $\left[\mathrm{CH}_{3} \mathrm{CH}_{2} \mathrm{CH}_{2}\right]^{+}: 43$ (61\%), $\left[\mathrm{CH}_{3} \mathrm{CH}_{2}\right]^{+}: 29$ (45\%); $\left[\mathrm{CH}_{3}\right]^{+}$: $15(37 \%)$.

Dibutyltin(IV)-di-stannoxane-di-3-phthalimido3(2-hydroxyphenyl) propanoate (2) (dimer)

Yield $72 \%$; mp $220{ }^{\circ} \mathrm{C}$; solubility: chloroform, THF; anal. calcd. (\%): C 41.5, H 6.5, N 3.4, found: $\mathrm{C} 41.3, \mathrm{H} 6.4, \mathrm{~N} 3.4$; FTIR $v_{\max } / \mathrm{cm}^{-1} 1608$ 


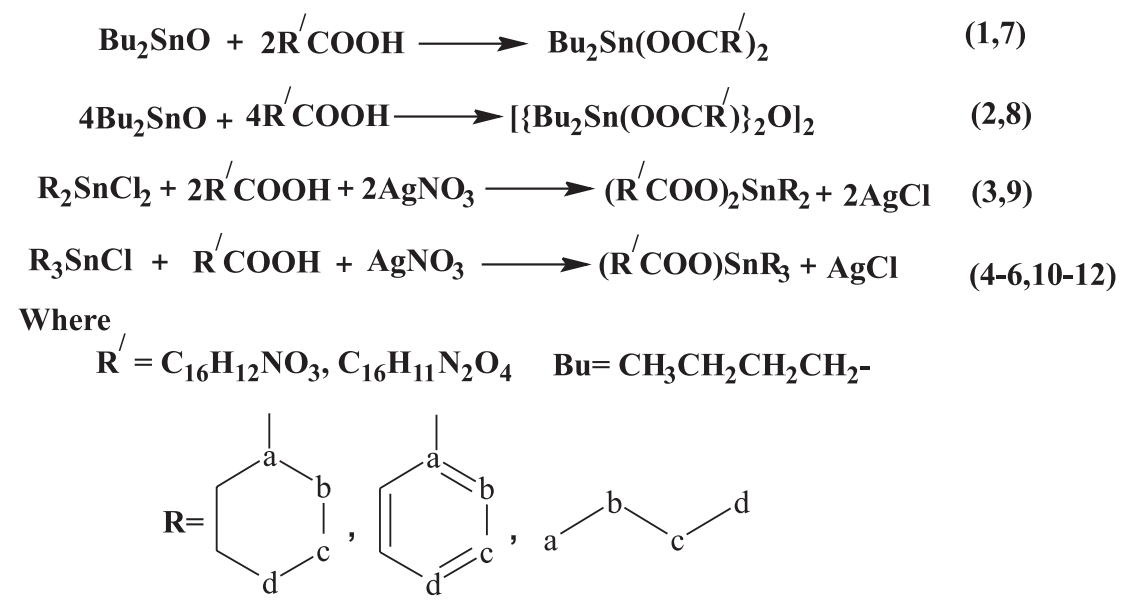

Scheme 2. Synthesis of organotin(IV) complexes.

$v(\mathrm{COO})_{\text {asym }}, 1455 v(\mathrm{COO})_{\text {sym }}, \Delta v 153,1727 v(\mathrm{~N}-\mathrm{CO})_{\text {asym }}$, $1660 v(\mathrm{~N}-\mathrm{CO})_{\mathrm{sym}}, 1230 v(\mathrm{C}-\mathrm{N}), 520 v(\mathrm{Sn}-\mathrm{C}), 502$ $v(\mathrm{Sn}-\mathrm{O}) ;{ }^{1} \mathrm{H}$ NMR $(400 \mathrm{MHz}, \mathrm{DMSO}) \delta 4.98(1 \mathrm{H}, \mathrm{s}, \mathrm{OH})$, $1.21(2 \mathrm{H}, \mathrm{d}, J 7.5 \mathrm{~Hz}, \mathrm{H} 2), 1.39(1 \mathrm{H}, \mathrm{t}, J 7.3 \mathrm{~Hz}, \mathrm{H} 3)$, $7.36(1 \mathrm{H}, \mathrm{d}, J 7.1 \mathrm{~Hz}, \mathrm{H} 6), 7.48(1 \mathrm{H}, \mathrm{d}, J 7.0 \mathrm{~Hz}, \mathrm{H} 7)$, $7.62(1 \mathrm{H}, \mathrm{d}, J 7.4 \mathrm{~Hz}, \mathrm{H} 10), 7.85(1 \mathrm{H}, \mathrm{d}, J 7.8 \mathrm{~Hz}, \mathrm{H} 11)$, $1.91(2 \mathrm{H}, \mathrm{t}, J 7.0 \mathrm{~Hz}, \mathrm{Ha}), 1.63(2 \mathrm{H}, \mathrm{m}, \mathrm{Hb}), 1.25(2 \mathrm{H}$, $\mathrm{m}, \mathrm{Hc}), 0.94$ (3H, t, $J 7.0 \mathrm{~Hz}, \mathrm{Hd}) ;{ }^{13} \mathrm{C}$ NMR $(100 \mathrm{MHz}$, DMSO) $\delta 170.42$ (C1), 45.53 (C2), 29.28 (C3), 123.45 (C4), 130.40 (C5), 132.15 (C6), 132.25 (C7), 163.51 (C8), 134.50 (C9), 136.78 (C10), 138.59 (C11), 27.10 (Ca), $23.19(\mathrm{Cb}), 21.63(\mathrm{Cc}), 12.12(\mathrm{Cd}) ;{ }^{119} \mathrm{Sn} \mathrm{NMR}\left(\mathrm{CH}_{3}\right)_{4} \mathrm{Sn}$ : 215.25; EI-MS $\mathrm{m} / z,\left[\left(\mathrm{C}_{4} \mathrm{H}_{9}\right)_{2} \mathrm{Sn}\left\{\mathrm{O}_{2} \mathrm{CC}_{16} \mathrm{H}_{12} \mathrm{NO}_{3}\right\}_{2}\right]^{+}$: $850(43 \%), \quad\left[\left(\mathrm{C}_{4} \mathrm{H}_{9}\right)_{2} \mathrm{SnO}_{2} \mathrm{C}\left\{\mathrm{C}_{16} \mathrm{H}_{12} \mathrm{NO}_{3}\right\}_{2}\right]^{+}$: $806(33 \%), \quad\left[\left(\mathrm{C}_{4} \mathrm{H}_{9}\right) \mathrm{Sn}\left\{\mathrm{O}_{2} \mathrm{CC}_{16} \mathrm{H}_{12} \mathrm{NO}_{3}\right\}_{2}\right]^{+}$: $793(59 \%), \quad\left[\left(\mathrm{C}_{4} \mathrm{H}_{9}\right) \mathrm{SnO} \mathrm{O}_{2} \mathrm{C}\left\{\mathrm{C}_{16} \mathrm{H}_{12} \mathrm{NO}_{3}\right\}_{2}\right]^{+}$: $749(52 \%), \quad\left[\left(\mathrm{C}_{4} \mathrm{H}_{9}\right) \mathrm{Sn}\left\{\mathrm{C}_{16} \mathrm{H}_{12} \mathrm{NO}_{3}\right\}_{2}\right]^{+}: 705$ (43\%), $\left[\left(\mathrm{C}_{4} \mathrm{H}_{9}\right)_{2} \mathrm{Sn}\left\{\mathrm{O}_{2} \mathrm{CC}_{16} \mathrm{H}_{12} \mathrm{NO}_{3}\right\}\right]^{+}: 541$ (35\%), $\left[\left(\mathrm{C}_{4} \mathrm{H}_{9}\right)_{2} \mathrm{Sn}\left\{\mathrm{C}_{16} \mathrm{H}_{12} \mathrm{NO}_{3}\right\}\right]^{+}: 497(47 \%),\left[\left(\mathrm{C}_{4} \mathrm{H}_{9}\right)\right.$ $\left.\mathrm{Sn}\left\{\mathrm{C}_{16} \mathrm{H}_{12} \mathrm{NO}_{3}\right\}\right]^{+}: 440(26 \%),\left[\mathrm{Sn}\left\{\mathrm{C}_{16} \mathrm{H}_{12} \mathrm{NO}_{3}\right\}\right]^{+}: 383$ (57\%), $\left[\mathrm{C}_{16} \mathrm{H}_{12} \mathrm{NO}_{3}\right]^{+}: 265$ (47\%), [Sn] $]^{+}: 119(100 \%)$, $\left[\mathrm{C}_{4} \mathrm{H}_{9}\right]^{+}: 57$ (63\%).

Dibutyltin(IV)-di-3-phthalimido-3(2-hydroxyphenyl) propanoate (3)

Yield 79\%; mp $166{ }^{\circ} \mathrm{C}$; solubility: chloroform, acetone, DMF, DMSO, THF; anal. calcd. (\%): C 41.3, H 6.4, N 5.8, found: C 41.5, H 6.2, N 5.10; FTIR $v_{\max } / \mathrm{cm}^{-1}$ $1635 v(\mathrm{COO})_{\text {asym }}, 1462 v(\mathrm{COO})_{\mathrm{sym}}, \Delta v 173,1720$ $v(\mathrm{~N}-\mathrm{CO})_{\text {asym }}, 1665 v(\mathrm{~N}-\mathrm{CO})_{\mathrm{sym}}, 1200 v(\mathrm{C}-\mathrm{N}), 515 v(\mathrm{Sn}-\mathrm{C})$, $505 v(\mathrm{Sn}-\mathrm{O}) ;{ }^{1} \mathrm{H}$ NMR $(400 \mathrm{MHz}, \mathrm{DMSO}) \delta 4.95(1 \mathrm{H}$, s, OH), $1.20(2 \mathrm{H}, \mathrm{d}, J 7.5 \mathrm{~Hz}, \mathrm{H} 2), 1.36(1 \mathrm{H}, \mathrm{t}, J 7.3 \mathrm{~Hz}$, H3), $7.28(1 \mathrm{H}, \mathrm{d}, J 7.1 \mathrm{~Hz}, \mathrm{H} 6), 7.48(1 \mathrm{H}, \mathrm{d}, J 7.0 \mathrm{~Hz}, \mathrm{H} 7)$, $7.53(1 \mathrm{H}, \mathrm{d}, J 7.4 \mathrm{~Hz}, \mathrm{H} 10), 7.67$ (1H, d, J 7.8 Hz, H11), $1.18(2 \mathrm{H}, \mathrm{t}, J 7.0 \mathrm{~Hz}, \mathrm{Ha}), 1.57(2 \mathrm{H}, \mathrm{m}, \mathrm{Hb}), 1.30(2 \mathrm{H}$, m, Hc), 0.95 (3H, t, J 7.0 Hz, Hd); ${ }^{13} \mathrm{C}$ NMR $(100 \mathrm{MHz}$,
DMSO) $\delta 177.65$ (C1), 43.35 (C2), 29.71 (C3), 121.34 (C4), 129.15 (C5), 130.35 (C6), 132.53 (C7), 164.75 (C8), 134.45 (C9), 135.91 (C10), 138.85 (C11), 21.56 (Ca), $25.35(\mathrm{Cb}), 26.89(\mathrm{Cc}), 12.95(\mathrm{Cd}) ;{ }^{119} \mathrm{Sn} \mathrm{NMR}\left(\mathrm{CH}_{3}\right)_{4} \mathrm{Sn}$ : 197.54; EI-MS $m / z,\left[\left(\mathrm{C}_{4} \mathrm{H}_{9}\right)_{2} \mathrm{Sn}\left\{\mathrm{O}_{2} \mathrm{CC}_{16} \mathrm{H}_{12} \mathrm{NO}_{3}\right\}_{2}\right]^{+}$: $850(41 \%), \quad\left[\left(\mathrm{C}_{4} \mathrm{H}_{9}\right)_{2} \mathrm{SnO}_{2} \mathrm{C}\left\{\mathrm{C}_{16} \mathrm{H}_{12} \mathrm{NO}_{3}\right\}_{2}\right]^{+}$: $806(32 \%), \quad\left[\left(\mathrm{C}_{4} \mathrm{H}_{9}\right) \mathrm{Sn}\left\{\mathrm{O}_{2} \mathrm{CC}_{16} \mathrm{H}_{12} \mathrm{NO}_{3}\right\}_{2}\right]^{+}$: $793(48 \%), \quad\left[\left(\mathrm{C}_{4} \mathrm{H}_{9}\right) \mathrm{SnO} \mathrm{O}_{2} \mathrm{C}\left\{\mathrm{C}_{16} \mathrm{H}_{12} \mathrm{NO}_{3}\right\}_{2}\right]^{+}$: $749(39 \%),\left[\left(\mathrm{C}_{4} \mathrm{H}_{9}\right) \mathrm{Sn}\left\{\mathrm{C}_{16} \mathrm{H}_{12} \mathrm{NO}_{3}\right\}_{2}\right]^{+}: 705$ (47\%), $\left[\left(\mathrm{C}_{4} \mathrm{H}_{9}\right)_{2} \mathrm{Sn}\left\{\mathrm{O}_{2} \mathrm{CC}_{16} \mathrm{H}_{12} \mathrm{NO}_{3}\right\}\right]^{+}: 541(35 \%)$, $\left[\left(\mathrm{C}_{4} \mathrm{H}_{9}\right)_{2} \mathrm{Sn}\left\{\mathrm{C}_{16} \mathrm{H}_{12} \mathrm{NO}_{3}\right\}\right]^{+}: 497(58 \%),\left[\left(\mathrm{C}_{4} \mathrm{H}_{9}\right)\right.$ $\left.\operatorname{Sn}\left\{\mathrm{C}_{16} \mathrm{H}_{12} \mathrm{NO}_{3}\right\}\right]^{+}: 440(30 \%),\left[\mathrm{Sn}\left\{\mathrm{C}_{16} \mathrm{H}_{12} \mathrm{NO}_{3}\right\}\right]^{+}: 383$ (100\%), $\left[\mathrm{C}_{16} \mathrm{H}_{12} \mathrm{NO}_{3}\right]^{+}: 265$ (43\%), [Sn] $: 119(74 \%)$, $\left[\mathrm{CH}_{3} \mathrm{CH}_{2} \mathrm{CH}_{2}\right]^{+}: 43(57 \%),\left[\mathrm{CH}_{3} \mathrm{CH}_{2}\right]^{+}: 29(62 \%) ;\left[\mathrm{CH}_{3}\right]^{+}$: $15(43 \%)$.

Tributyltin(IV)-3-phthalimido-3(2-hydroxyphenyl) propanoate (4)

Yield $85 \%$; mp $105^{\circ} \mathrm{C}$; solubility: chloroform, acetone, DMF, DMSO, THF; anal. calcd. (\%): C 48.2, H 7.9, N 3.3, found: C 48.2, H 7.8, N 3.1; FTIR $v_{\max } / \mathrm{cm}^{-1} 1610$ $v(\mathrm{COO})_{\text {asym }}, 1451 v(\mathrm{COO})_{\text {sym }}, \Delta v 159,1725 v(\mathrm{~N}-\mathrm{CO})_{\text {asym }}$, $1620 v(\mathrm{~N}-\mathrm{CO})_{\text {sym }}, 1150 v(\mathrm{C}-\mathrm{N}), 517 v(\mathrm{Sn}-\mathrm{C}), 505$ $v(\mathrm{Sn}-\mathrm{O}) ;{ }^{1} \mathrm{H}$ NMR $(400 \mathrm{MHz}, \mathrm{DMSO}) \delta 4.89(1 \mathrm{H}, \mathrm{s}, \mathrm{OH})$, $1.28(2 \mathrm{H}, \mathrm{d}, J 7.5 \mathrm{~Hz}, \mathrm{H} 2), 1.41(1 \mathrm{H}, \mathrm{t}, J 7.3 \mathrm{~Hz}, \mathrm{H} 3)$, $7.76(1 \mathrm{H}, \mathrm{d}, J 7.1 \mathrm{~Hz}, \mathrm{H} 6), 7.79(1 \mathrm{H}, \mathrm{d}, J 7.0 \mathrm{~Hz}, \mathrm{H} 7)$, $7.89(1 \mathrm{H}, \mathrm{d}, J 7.4 \mathrm{~Hz}, \mathrm{H} 10), 7.96(1 \mathrm{H}, \mathrm{d}, J 7.8 \mathrm{~Hz}, \mathrm{H} 11)$, $1.76(2 \mathrm{H}, \mathrm{t}, J 7.0 \mathrm{~Hz}, \mathrm{Ha}), 1.30(2 \mathrm{H}, \mathrm{m}, \mathrm{Hb}), 1.25(2 \mathrm{H}$, m, Hc), 0.90 (3H, t, J 7.0 Hz, Hd); ${ }^{13} \mathrm{C}$ NMR (100 MHz, DMSO) $\delta 176.93$ (C1), 47.45 (C2), 24.72 (C3), 123.45 (C4), 129.01 (C5), 131.40 (C6), 132.95 (C7), 165.05 (C8), 134 (C9), 135.91 (C10), 138.54 (C11), 23.50 (Ca), $25.12(\mathrm{Cb}), 26.30(\mathrm{Cc}), 13.45(\mathrm{Cd}) ;{ }^{119} \mathrm{Sn} \mathrm{NMR}\left(\mathrm{CH}_{3}\right)_{4} \mathrm{Sn}$ : 123.76; EI-MS m/z, $\left[\left(\mathrm{C}_{4} \mathrm{H}_{9}\right)_{3} \mathrm{Sn}\left\{\mathrm{O}_{2} \mathrm{CC}_{16} \mathrm{H}_{12} \mathrm{NO}_{3}\right\}\right]^{+}$: $598(31 \%),\left[\left(\mathrm{C}_{4} \mathrm{H}_{9}\right)_{2} \mathrm{Sn}\left\{\mathrm{O}_{2} \mathrm{CC}_{16} \mathrm{H}_{12} \mathrm{NO}_{3}\right\}\right]^{+}: 541$ (100\%), $\left[\left(\mathrm{C}_{4} \mathrm{H}_{9}\right)_{2} \mathrm{Sn}\left\{\mathrm{C}_{16} \mathrm{H}_{12} \mathrm{NO}_{3}\right\}\right]^{+}: 497(60 \%)$, 
$\left[\left(\mathrm{C}_{4} \mathrm{H}_{9}\right) \mathrm{Sn}\left\{\mathrm{C}_{16} \mathrm{H}_{12} \mathrm{NO}_{3}\right\}\right]^{+}: 440(28 \%),\left[\left(\mathrm{C}_{4} \mathrm{H}_{9}\right)_{3} \mathrm{Sn}\right]^{+}: 289$ (30\%), $\left[\mathrm{C}_{16} \mathrm{H}_{12} \mathrm{NO}_{3}\right]^{+}: 265(43 \%),\left[\left(\mathrm{C}_{4} \mathrm{H}_{9}\right)_{2} \mathrm{Sn}\right]^{+}: 232(21 \%)$, $\left[\left(\mathrm{C}_{4} \mathrm{H}_{9}\right) \mathrm{Sn}\right]^{+}: 175(17 \%),[\mathrm{Sn}]^{+}: 119(75 \%),\left[\mathrm{CH}_{3} \mathrm{CH}_{2} \mathrm{CH}_{2}\right]^{+}$: $43(44 \%),\left[\mathrm{CH}_{3} \mathrm{CH}_{2}\right]^{+}: 29(56 \%) ;\left[\mathrm{CH}_{3}\right]^{+}: 15(46 \%)$.

Triphenyltin(IV)-3-phthalimido-3(2-hydroxyphenyl) propanoate (5)

Yield $78 \%$; mp $126{ }^{\circ} \mathrm{C}$; solubility: acetone, DMF, DMSO; anal. calcd. (\%): C 56.9, H 4.7, N 2.7, found: C 56.7, H 4.5, N 2.7; FTIR $v_{\max } / \mathrm{cm}^{-1} 1656 v(\mathrm{COO})_{\text {asym }}, 1471$ $v(\mathrm{COO})_{\mathrm{sym}}, \Delta v 185,1728 v(\mathrm{~N}-\mathrm{CO})_{\text {asym }}, 1670 v(\mathrm{~N}-\mathrm{CO})_{\text {sym }}$, $1220 v(\mathrm{C}-\mathrm{N}), 547 v(\mathrm{Sn}-\mathrm{C}), 512 v(\mathrm{Sn}-\mathrm{O}) ;{ }^{1} \mathrm{H}$ NMR (400 MHz, DMSO) $\delta 5.09(1 \mathrm{H}, \mathrm{s}, \mathrm{OH}), 1.35(2 \mathrm{H}, \mathrm{d}, J 7.5 \mathrm{~Hz}$, H2), $1.41(1 \mathrm{H}, \mathrm{t}, J 7.3 \mathrm{~Hz}, \mathrm{H} 3), 7.45(1 \mathrm{H}, \mathrm{d}, J 7.1 \mathrm{~Hz}, \mathrm{H} 6)$, $7.51(1 \mathrm{H}, \mathrm{d}, J 7.0 \mathrm{~Hz}, \mathrm{H} 7), 7.65(1 \mathrm{H}, \mathrm{d}, J 7.4 \mathrm{~Hz}, \mathrm{H} 10)$, $7.84(1 \mathrm{H}, \mathrm{d}, J 7.8 \mathrm{~Hz}, \mathrm{H} 11), 7.64(1 \mathrm{H}, \mathrm{t}, J 7.0 \mathrm{~Hz}, \mathrm{Hb})$, $7.74(1 \mathrm{H}, \mathrm{m}, \mathrm{Hc}), 7.79(1 \mathrm{H}, \mathrm{t}, J 7.0 \mathrm{~Hz}, \mathrm{Hd}) ;{ }^{13} \mathrm{C} \mathrm{NMR}$ (100 MHz, DMSO) $\delta 175.92(\mathrm{C} 1), 42.75(\mathrm{C} 2), 29.52(\mathrm{C} 3)$, 124.35 (C4), 125.65 (C5), 129.45 (C6), 131.83 (C7), 163.51 (C8), 134.15 (C9), 135.97 (C10), 137.02 (C11), 137.61 (Ca), $139.54(\mathrm{Cb}), 141.18(\mathrm{Cc}), 143.39(\mathrm{Cd}) ;{ }^{119} \mathrm{Sn}$ NMR $\left(\mathrm{CH}_{3}\right)_{4} \mathrm{Sn}: 99.28 ; \mathrm{EI}-\mathrm{MS} m / z,\left[\left(\mathrm{C}_{6} \mathrm{H}_{5}\right)_{3} \mathrm{Sn}\left\{\mathrm{O}_{2} \mathrm{CC}_{16} \mathrm{H}_{12} \mathrm{NO}_{3}\right\}\right]^{+}$: $658(30 \%),\left[\left(\mathrm{C}_{6} \mathrm{H}_{5}\right)_{2} \mathrm{Sn}\left\{\mathrm{O}_{2} \mathrm{CC}_{16} \mathrm{H}_{12} \mathrm{NO}_{3}\right\}\right]^{+}: 581$ (43\%), $\left[\left(\mathrm{C}_{6} \mathrm{H}_{5}\right)_{2} \mathrm{Sn}\left\{\mathrm{C}_{16} \mathrm{H}_{12} \mathrm{NO}_{3}\right\}\right]^{+}: 537(61 \%),\left[\left(\mathrm{C}_{6} \mathrm{H}_{5}\right)\right.$ $\left.\mathrm{Sn}\left\{\mathrm{C}_{16} \mathrm{H}_{12} \mathrm{NO}_{3}\right\}\right]^{+}: 460(58 \%),\left[\left(\mathrm{C}_{6} \mathrm{H}_{5}\right)_{3} \mathrm{Sn}\right]^{+}: 349(33 \%)$, $\left[\left(\mathrm{C}_{6} \mathrm{H}_{5}\right)_{2} \mathrm{Sn}\right]^{+}: 272(28 \%),\left[\mathrm{C}_{16} \mathrm{H}_{12} \mathrm{NO}_{3}\right]^{+}: 265(39 \%),\left[\left(\mathrm{C}_{6} \mathrm{H}_{5}\right)\right.$ $\mathrm{Sn}]^{+}: 195$ (100\%), [Sn] : 119 (74\%), $\left[\mathrm{C}_{6} \mathrm{H}_{5}\right]^{+}: 77$ (49\%).

Tricyclohexyltin(IV)-3-phthalimido-3(2-hydroxyphenyl) propanoate (6)

Yield 76\%; mp $112{ }^{\circ} \mathrm{C}$; solubility: chloroform, acetone, DMSO, THF; anal. calcd. (\%): C 54.8, H 7.7, N 2.6, found: C 54.7, H 7.8, N 2.6; FTIR $v_{\max } / \mathrm{cm}^{-1} 1645$ $v(\mathrm{COO})_{\text {asym }}, 1490 v(\mathrm{COO})_{\mathrm{sym}}, \Delta v 155,1720 v(\mathrm{~N}-\mathrm{CO})_{\text {asym }}$, $1650 v(\mathrm{~N}-\mathrm{CO})_{\text {sym }}, 1050 v(\mathrm{C}-\mathrm{N}), 540 v(\mathrm{Sn}-\mathrm{C}), v(\mathrm{Sn}-\mathrm{O})$ 518; ${ }^{1} \mathrm{H}$ NMR (400 MHz, DMSO) $\delta 5.08(1 \mathrm{H}, \mathrm{s}, \mathrm{OH})$, $1.23(2 \mathrm{H}, \mathrm{d}, J 7.5 \mathrm{~Hz}, \mathrm{H} 2), 1.34(1 \mathrm{H}, \mathrm{t}, J 7.3 \mathrm{~Hz}, \mathrm{H} 3)$, $7.59(1 \mathrm{H}, \mathrm{d}, J 7.1 \mathrm{~Hz}, \mathrm{H} 6), 7.66(1 \mathrm{H}, \mathrm{d}, J 7.0 \mathrm{~Hz}, \mathrm{H} 7)$, 8.06 (1H, d, J 7.4 Hz, H10), 8.15 (1H, d, J 7.8 Hz, H11), $1.81(2 \mathrm{H}, \mathrm{t}, J 7.0 \mathrm{~Hz}, \mathrm{Ha}), 1.55(2 \mathrm{H}, \mathrm{m}, \mathrm{Hb}) 1.35(2 \mathrm{H}$, $\mathrm{m}, \mathrm{Hc}), 1.14$ (2H, m, Hd); ${ }^{13} \mathrm{C}$ NMR (100 MHz, DMSO)

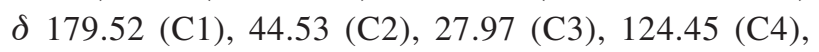
127.68 (C5), 131.45 (C6), 132.95 (C7), 164.95 (C8), 135.50 (C9), 136.42 (C10), 137.13 (C11), 22.69 (Ca), $28.89(\mathrm{Cb}), 26.44(\mathrm{Cc}), 12.55(\mathrm{Cd}) ;{ }^{119} \mathrm{Sn} \mathrm{NMR}\left(\mathrm{CH}_{3}\right)_{4} \mathrm{Sn}$ : 9.15; EI-MS m/z, [( $\left.\left.\mathrm{C}_{6} \mathrm{H}_{11}\right)_{3} \mathrm{Sn}\left\{\mathrm{O}_{2} \mathrm{CC}_{16} \mathrm{H}_{12} \mathrm{NO}_{3}\right\}\right]^{+}: 676$ $(30 \%),\left[\left(\mathrm{C}_{6} \mathrm{H}_{11}\right)_{2} \mathrm{Sn}\left\{\mathrm{O}_{2} \mathrm{CC}_{16} \mathrm{H}_{12} \mathrm{NO}_{3}\right\}\right]^{+}: 593$ (43\%), $\left[\left(\mathrm{C}_{6} \mathrm{H}_{11}\right)_{2} \mathrm{Sn}\left\{\mathrm{C}_{16} \mathrm{H}_{12} \mathrm{NO}_{3}\right\}\right]^{+}: 549(62 \%),\left[\left(\mathrm{C}_{6} \mathrm{H}_{11}\right)\right.$ $\left.\mathrm{Sn}\left\{\mathrm{C}_{16} \mathrm{H}_{12} \mathrm{NO}_{3}\right\}\right]^{+}: 466$ (59\%), [( $\left.\left.\mathrm{C}_{6} \mathrm{H}_{11}\right)_{3} \mathrm{Sn}\right]^{+}: 367$ (34\%), $\left[\left(\mathrm{C}_{6} \mathrm{H}_{11}\right)_{2} \mathrm{Sn}\right]^{+}: 284(29 \%),\left[\mathrm{C}_{16} \mathrm{H}_{12} \mathrm{NO}_{3}\right]^{+}: 265(39 \%),\left[\left(\mathrm{C}_{6} \mathrm{H}_{11}\right)\right.$ $\mathrm{Sn}]^{+}: 201(100 \%),[\mathrm{Sn}]^{+}: 119(69 \%),\left[\mathrm{C}_{6} \mathrm{H}_{11}\right]^{+}: 83(46 \%)$.
Dibutyltin(IV)-di-3-phthalimido-3(2-nitrophenyl) propanoate (7) (monomer)

Yield 78\%; mp $204{ }^{\circ} \mathrm{C}$; solubility: chloroform, DMF, DMSO, THF; anal. calcd. (\%): C 43.2, H 6.4, N 5.9, found: C 43.3, H 6.2, N 5.10; FTIR $v_{\max } / \mathrm{cm}^{-1} 1650 v(\mathrm{COO})_{\text {asym }}$, $1440 v(\mathrm{COO})_{\mathrm{sym}}, \Delta v 170,1726 v(\mathrm{~N}-\mathrm{CO})_{\mathrm{asym}}, 1670$ $v(\mathrm{~N}-\mathrm{CO})_{\text {sym }}, 1180 v(\mathrm{C}-\mathrm{N}), 515 v(\mathrm{Sn}-\mathrm{C}), 505 v(\mathrm{Sn}-\mathrm{O})$; ${ }^{1} \mathrm{H}$ NMR (400 MHz, DMSO) $\delta 1.29$ (2H, d, J 7.3 Hz, H2), $1.41(1 \mathrm{H}, \mathrm{t}, J 7.5 \mathrm{~Hz}, \mathrm{H} 3), 7.28$ (1H, d, J 7.3 Hz, H6), 7.48 $(1 \mathrm{H}, \mathrm{d}, J 7.1 \mathrm{~Hz}, \mathrm{H} 7), 7.53(1 \mathrm{H}, \mathrm{d}, J 7.5 \mathrm{~Hz}, \mathrm{H} 10) 7.67(1 \mathrm{H}$, d, J 7.1 Hz, H11), $1.16(2 \mathrm{H}, \mathrm{t}, J 7.0 \mathrm{~Hz}, \mathrm{Ha}), 1.54(2 \mathrm{H}, \mathrm{m}$, $\mathrm{Hb}), 1.23$ (2H, m, Hc), 0.91 (3H, t, J 7.0 Hz, Hd); ${ }^{13} \mathrm{C} \mathrm{NMR}$ (100 MHz, DMSO) $\delta 170.35$ (C1), 42.25 (C2), 29.45 (C3), 123.75 (C4), 125.90 (C5), 129.19 (C6), 131.40 (C7), 163.65 (C8), 136.45 (C9), 138.90 (C10), 139.01 (C11), 26.45 (Ca), $23.35(\mathrm{Cb}), 21.45(\mathrm{Cc}), 12.98(\mathrm{Cd}) ;{ }^{119} \mathrm{Sn} \mathrm{NMR}\left(\mathrm{CH}_{3}\right)_{4} \mathrm{Sn}$ : 185.58; EI-MS m/z, [( $\left.\left(\mathrm{C}_{4} \mathrm{H}_{9}\right)_{2} \mathrm{Sn}\left\{\mathrm{O}_{2} \mathrm{CC}_{16} \mathrm{H}_{11} \mathrm{~N}_{2} \mathrm{O}_{4}\right\}_{2}\right]^{+}$: $910(36 \%),\left[\left(\mathrm{C}_{4} \mathrm{H}_{9}\right) \mathrm{Sn}\left\{\mathrm{O}_{2} \mathrm{CC}_{16} \mathrm{H}_{11} \mathrm{~N}_{2} \mathrm{O}_{4}\right\}_{2}\right]^{+}: 853$ (24\%), $\left[\left(\mathrm{C}_{4} \mathrm{H}_{9}\right) \mathrm{SnO}_{2} \mathrm{C}\left\{\mathrm{C}_{16} \mathrm{H}_{11} \mathrm{~N}_{2} \mathrm{O}_{4}\right\}_{2}\right]^{+}: 809(100 \%),\left[\left(\mathrm{C}_{4} \mathrm{H}_{9}\right) \mathrm{Sn}\right.$ $\left.\left\{\mathrm{C}_{16} \mathrm{H}_{11} \mathrm{~N}_{2} \mathrm{O}_{4}\right\}_{2}\right]^{+}: 765(47 \%),\left[\left(\mathrm{C}_{4} \mathrm{H}_{9}\right)_{2} \mathrm{Sn}\left\{\mathrm{O}_{2} \mathrm{CC}_{16} \mathrm{H}_{11} \mathrm{~N}_{2} \mathrm{O}_{4}\right\}\right]^{+}$: $571(29 \%),\left[\left(\mathrm{C}_{4} \mathrm{H}_{9}\right)_{2} \mathrm{Sn}\left\{\mathrm{C}_{16} \mathrm{H}_{11} \mathrm{~N}_{2} \mathrm{O}_{4}\right\}\right]^{+}: 527(58 \%),\left[\left(\mathrm{C}_{4} \mathrm{H}_{9}\right)\right.$ $\left.\mathrm{Sn}\left\{\mathrm{C}_{16} \mathrm{H}_{11} \mathrm{~N}_{2} \mathrm{O}_{4}\right\}\right]^{+}: 470(28 \%),\left[\mathrm{Sn}\left\{\mathrm{C}_{16} \mathrm{H}_{11} \mathrm{~N}_{2} \mathrm{O}_{4}\right\}\right]^{+}: 413$ (69\%), $\left[\mathrm{C}_{16} \mathrm{H}_{11} \mathrm{~N}_{2} \mathrm{O}_{4}\right]^{+}: 295$ (39\%), [Sn] : 119 (75\%), $\left[\mathrm{CH}_{3} \mathrm{CH}_{2} \mathrm{CH}_{2}\right]^{+}: 43(68 \%),\left[\mathrm{CH}_{3} \mathrm{CH}_{2}\right]^{+}: 29(56 \%) ;\left[\mathrm{CH}_{3}\right]^{+}$: $15(46 \%)$.

Dibutyltin(IV)-di-stannoxane-di-3-phthalimido-3(2nitrophenyl) propanoate (8) (dimer)

Yield 79\%; mp $240{ }^{\circ} \mathrm{C}$; solubility: chloroform, THF; anal. calcd. (\%): C 42.3, H 6.5, N 3.6, found: C 42.1, H 6.4, N 3.4; FTIR $v_{\max } / \mathrm{cm}^{-1} 1628 v(\mathrm{COO})_{\text {asym }}, 1475 v(\mathrm{COO})_{\mathrm{sym}}$, $\Delta v 153,1730 v(\mathrm{~N}-\mathrm{CO})_{\text {asym }}, 1670 v(\mathrm{~N}-\mathrm{CO})_{\text {sym }}, 1210 v(\mathrm{C}-\mathrm{N})$, $550 v(\mathrm{Sn}-\mathrm{C}), 510 v(\mathrm{Sn}-\mathrm{O}) ;{ }^{1} \mathrm{H}$ NMR $(400 \mathrm{MHz}, \mathrm{DMSO})$ $\delta 1.27(2 \mathrm{H}, \mathrm{d}, J 7.3 \mathrm{~Hz}, \mathrm{H} 2), 1.32(1 \mathrm{H}, \mathrm{t}, J 7.5 \mathrm{~Hz}, \mathrm{H} 3)$, 7.65 (1H, d, J $7.3 \mathrm{~Hz}, \mathrm{H} 6), 7.79(1 \mathrm{H}, \mathrm{d}, J 7.1 \mathrm{~Hz}, \mathrm{H} 7)$, 7.89 (1H, d, J $7.5 \mathrm{~Hz}, \mathrm{H} 10), 7.95$ (1H, d, J 7.1 Hz, H11), $1.93(2 \mathrm{H}, \mathrm{t}, J 7.0 \mathrm{~Hz}, \mathrm{Ha}), 1.62(2 \mathrm{H}, \mathrm{m}, \mathrm{Hb}), 1.14(2 \mathrm{H}$, m, Hc), 0.95 (3H, t, J $7.0 \mathrm{~Hz}, \mathrm{Hd}) ;{ }^{13} \mathrm{C} \mathrm{NMR} \mathrm{(100} \mathrm{MHz,}$ DMSO) $\delta 179.45$ (C1), 43.33 (C2), 28.75 (C3), 125.56 (C4), 129.98 (C5), 131.36 (C6), 133.15 (C7), 163.91 (C8), 134.45 (C9), 137.25 (C10), 138.80 (C11), 27.65 (Ca), $26.78(\mathrm{Cb}), 25.40(\mathrm{Cc}), 13.15(\mathrm{Cd}) ;{ }^{119} \mathrm{Sn} \mathrm{NMR}\left(\mathrm{CH}_{3}\right)_{4} \mathrm{Sn}:$ 216.24; EI-MS m/z, [( $\left.\left(\mathrm{C}_{4} \mathrm{H}_{9}\right)_{2} \mathrm{Sn}\left\{\mathrm{O}_{2} \mathrm{CC}_{16} \mathrm{H}_{11} \mathrm{~N}_{2} \mathrm{O}_{4}\right\}_{2}\right]^{+}$: 910 (19\%), $\left[\left(\mathrm{C}_{4} \mathrm{H}_{9}\right) \mathrm{Sn}\left\{\mathrm{O}_{2} \mathrm{CC}_{16} \mathrm{H}_{11} \mathrm{~N}_{2} \mathrm{O}_{4}\right\}_{2}\right]^{+}: 853$ (24\%), [ $\left.\left(\mathrm{C}_{4} \mathrm{H}_{9}\right) \mathrm{SnO}_{2} \mathrm{C}\left\{\mathrm{C}_{16} \mathrm{H}_{11} \mathrm{~N}_{2} \mathrm{O}_{4}\right\}_{2}\right]^{+}: 809$ (70\%), $\left[\left(\mathrm{C}_{4} \mathrm{H}_{9}\right) \mathrm{Sn}\left\{\mathrm{C}_{16} \mathrm{H}_{11} \mathrm{~N}_{2} \mathrm{O}_{4}\right\}_{2}\right]^{+}: 765$ (47\%), $\left[\left(\mathrm{C}_{4} \mathrm{H}_{9}\right)_{2} \mathrm{Sn}\left\{\mathrm{O}_{2} \mathrm{CC}_{16} \mathrm{H}_{11} \mathrm{~N}_{2} \mathrm{O}_{4}\right\}\right]^{+}: 571(34 \%)$, $\left[\left(\mathrm{C}_{4} \mathrm{H}_{9}\right)_{2} \mathrm{Sn}\left\{\mathrm{C}_{16} \mathrm{H}_{11} \mathrm{~N}_{2} \mathrm{O}_{4}\right\}\right]^{+}: 527(60 \%),\left[\left(\mathrm{C}_{4} \mathrm{H}_{9}\right)\right.$ $\left.\mathrm{Sn}\left\{\mathrm{C}_{16} \mathrm{H}_{11} \mathrm{~N}_{2} \mathrm{O}_{4}\right\}\right]^{+}: 470(100 \%),\left[\operatorname{Sn}\left\{\mathrm{C}_{16} \mathrm{H}_{11} \mathrm{~N}_{2} \mathrm{O}_{4}\right\}\right]^{+}: 413$ (69\%), $\left[\mathrm{C}_{16} \mathrm{H}_{11} \mathrm{~N}_{2} \mathrm{O}_{4}\right]^{+}: 295(57 \%),[\mathrm{Sn}]^{+}: 119(73 \%),\left[\mathrm{C}_{4} \mathrm{H}_{9}\right]^{+}$: 57 (69\%). 
Dibutyltin(IV)-di-3-phthalimido-3(2-nitrophenyl) propanoate (9)

Yield $75 \%$; mp $175^{\circ} \mathrm{C}$; solubility: chloroform, acetone, DMF, DMSO, THF; anal. calcd. (\%): C 41.3, H 6.3, N 5.9, found: C41.3, H6.2, N 5.10; FTIR $v_{\max } / \mathrm{cm}^{-1} 1645 v(\mathrm{COO})_{\text {asym }}$, $1472 v(\mathrm{COO})_{\mathrm{sym}}, \Delta v 173,1735 v(\mathrm{~N}-\mathrm{CO})_{\text {asym }}, 1675$ $v(\mathrm{~N}-\mathrm{CO})_{\text {sym }}, 1080 v(\mathrm{C}-\mathrm{N}), 520 v(\mathrm{Sn}-\mathrm{C}), 502 v(\mathrm{Sn}-\mathrm{O})$; ${ }^{1} \mathrm{H}$ NMR (400 MHz, DMSO) $\delta 1.30(2 \mathrm{H}, \mathrm{d}, J 7.3 \mathrm{~Hz}, \mathrm{H} 2)$, $1.40(1 \mathrm{H}, \mathrm{t}, J 7.5 \mathrm{~Hz}, \mathrm{H} 3), 7.64(1 \mathrm{H}, \mathrm{d}, J 7.3 \mathrm{~Hz}, \mathrm{H} 6), 7.66$ $(1 \mathrm{H}, \mathrm{d}, J 7.1 \mathrm{~Hz}, \mathrm{H} 7), 7.69(1 \mathrm{H}, \mathrm{d}, J 7.5 \mathrm{~Hz}, \mathrm{H} 10), 7.79(1 \mathrm{H}$, d, J 7.1 Hz, H11), 1.15 (2H, t, J 7.0 Hz, Ha), $1.53(2 \mathrm{H}, \mathrm{m}$, $\mathrm{Hb}), 1.32(2 \mathrm{H}, \mathrm{m}, \mathrm{Hc}), 0.92(3 \mathrm{H}, \mathrm{t}, J 7.0 \mathrm{~Hz}, \mathrm{Hd}) ;{ }^{13} \mathrm{C} \mathrm{NMR}$ (100 MHz, DMSO) $\delta 176.75$ (C1), 43.55 (C2), 30.15 (C3), 123.45 (C4), 125.50 (C5), 126.75 (C6), 132.20 (C7), 164.15 (C8), 133.85 (C9), 135.25 (C10), 138.91 (C11), 29.65 (Ca), $27.50(\mathrm{Cb}), 26.93(\mathrm{Cc}), 12.75(\mathrm{Cd}) ;{ }^{119} \mathrm{Sn} \mathrm{NMR}\left(\mathrm{CH}_{3}\right)_{4} \mathrm{Sn}$ : 195.55; EI-MS $m / z,\left[\left(\mathrm{C}_{4} \mathrm{H}_{9}\right)_{2} \mathrm{Sn}\left\{\mathrm{O}_{2} \mathrm{CC}_{16} \mathrm{H}_{11} \mathrm{~N}_{2} \mathrm{O}_{4}\right\}_{2}\right]^{+}$: $910(37 \%),\left[\left(\mathrm{C}_{4} \mathrm{H}_{9}\right) \mathrm{Sn}\left\{\mathrm{O}_{2} \mathrm{CC}_{16} \mathrm{H}_{11} \mathrm{~N}_{2} \mathrm{O}_{4}\right\}_{2}\right]^{+}: 853$ (23\%), [( $\left.\left.\mathrm{C}_{4} \mathrm{H}_{9}\right) \mathrm{SnO}_{2} \mathrm{C}\left\{\mathrm{C}_{16} \mathrm{H}_{11} \mathrm{~N}_{2} \mathrm{O}_{4}\right\}_{2}\right]^{+}: 809$ (69\%), $\left[\left(\mathrm{C}_{4} \mathrm{H}_{9}\right) \mathrm{Sn}\left\{\mathrm{C}_{16} \mathrm{H}_{11} \mathrm{~N}_{2} \mathrm{O}_{4}\right\}_{2}\right]^{+}: 765(47 \%),\left[\left(\mathrm{C}_{4} \mathrm{H}_{9}\right)_{2} \mathrm{Sn}\right.$ $\left.\left\{\mathrm{O}_{2} \mathrm{CC}_{16} \mathrm{H}_{11} \mathrm{~N}_{2} \mathrm{O}_{4}\right\}\right]^{+}: 571(30 \%),\left[\left(\mathrm{C}_{4} \mathrm{H}_{9}\right)_{2} \mathrm{Sn}\left\{\mathrm{C}_{16} \mathrm{H}_{11} \mathrm{~N}_{2} \mathrm{O}_{4}\right\}\right]^{+}$: $527(58 \%),\left[\left(\mathrm{C}_{4} \mathrm{H}_{9}\right) \mathrm{Sn}\left\{\mathrm{C}_{16} \mathrm{H}_{11} \mathrm{~N}_{2} \mathrm{O}_{4}\right\}\right]^{+}: 470(28 \%)$, $\left[\mathrm{Sn}\left\{\mathrm{C}_{16} \mathrm{H}_{11} \mathrm{~N}_{2} \mathrm{O}_{4}\right\}\right]^{+}: 413(69 \%),\left[\mathrm{C}_{16} \mathrm{H}_{11} \mathrm{~N}_{2} \mathrm{O}_{4}\right]^{+}: 295(100 \%)$, [Sn] : 119 (74\%), [ $\left[\mathrm{CH}_{3} \mathrm{CH}_{2} \mathrm{CH}_{2}\right]^{+}: 43(68 \%),\left[\mathrm{CH}_{3} \mathrm{CH}_{2}\right]^{+}$: $29(56 \%) ;\left[\mathrm{CH}_{3}\right]^{+}: 15(46 \%)$.

Tributyltin(IV)-3-phthaimido-3(2-nitrophenyl) propanoate (10)

Yield $75 \%$; mp $110{ }^{\circ} \mathrm{C}$; solubility: acetone, DMF, DMSO, THF; anal. calcd. (\%): C 48.3, H 7.9, N 3.2, found: C 48.2, H 7.8, N 3.1; FTIR $v_{\max } / \mathrm{cm}^{-1} 1664 v(\mathrm{COO})_{\text {asym }}$, $1510 v(\mathrm{COO})_{\text {sym }}, \Delta v 154,1750 v(\mathrm{~N}-\mathrm{CO})_{\text {asym }}, 1675$ $v(\mathrm{~N}-\mathrm{CO})_{\text {sym }}, 1050 v(\mathrm{C}-\mathrm{N}), 540 v(\mathrm{Sn}-\mathrm{C}), 520 v(\mathrm{Sn}-\mathrm{O})$; ${ }^{1} \mathrm{H}$ NMR (400 MHz, DMSO) $\delta 1.25(2 \mathrm{H}, \mathrm{d}, J 7.3 \mathrm{~Hz}, \mathrm{H} 2)$, $1.38(1 \mathrm{H}, \mathrm{t}, J 7.5 \mathrm{~Hz}, \mathrm{H} 3), 7.86(1 \mathrm{H}, \mathrm{d}, J 7.3 \mathrm{~Hz}, \mathrm{H} 6), 7.89$ $(1 \mathrm{H}, \mathrm{d}, J 7.1 \mathrm{~Hz}, \mathrm{H} 7), 7.90(1 \mathrm{H}, \mathrm{d}, J 7.5 \mathrm{~Hz}, \mathrm{H} 10), 7.98(1 \mathrm{H}$, d, J 7.1 Hz, H11), $1.56(2 \mathrm{H}, \mathrm{t}, J 7.0 \mathrm{~Hz}, \mathrm{Ha}), 1.36(2 \mathrm{H}, \mathrm{m}$, $\mathrm{Hb}), 1.27$ (2H, m, Hc), 0.89 (3H, t, J 7.0 Hz, Hd); ${ }^{13} \mathrm{C} \mathrm{NMR}$ (100 MHz, DMSO) $\delta 170.93$ (C1), 45.50 (C2), 29.72 (C3), 125.45 (C4), 127.28 (C5), 129.35 (C6), 131.60 (C7), 163.55 (C8), 135.50 (C9), 137.91 (C10), 138.56 (C11), $28.45(\mathrm{Ca}), 27.53(\mathrm{Cb}), 26.35(\mathrm{Cc}), 12.85(\mathrm{Cd})$; ${ }^{119} \mathrm{Sn}$ NMR $\left(\mathrm{CH}_{3}\right)_{4} \mathrm{Sn}: 135.79$; EI-MS $\mathrm{m} / z$, $\left[\left(\mathrm{C}_{4} \mathrm{H}_{9}\right)_{3} \mathrm{Sn}\left\{\mathrm{O}_{2} \mathrm{CC}_{16} \mathrm{H}_{11} \mathrm{~N}_{2} \mathrm{O}_{4}\right\}\right]^{+}: 628(47 \%),\left[\left(\mathrm{C}_{4} \mathrm{H}_{9}\right)_{2} \mathrm{Sn}\right.$ $\left.\left\{\mathrm{O}_{2} \mathrm{CC}_{16} \mathrm{H}_{11} \mathrm{~N}_{2} \mathrm{O}_{4}\right\}\right]^{+}: 571(100 \%), \quad\left[\left(\mathrm{C}_{4} \mathrm{H}_{9}\right)_{2} \mathrm{Sn}\right.$ $\left.\left\{\mathrm{C}_{16} \mathrm{H}_{11} \mathrm{~N}_{2} \mathrm{O}_{4}\right\}\right]^{+}: 527$ (58\%), [( $\left.\left.\mathrm{C}_{4} \mathrm{H}_{9}\right) \operatorname{Sn}\left\{\mathrm{C}_{16} \mathrm{H}_{11} \mathrm{~N}_{2} \mathrm{O}_{4}\right\}\right]^{+}$: 470 (25\%), $\left[\mathrm{C}_{16} \mathrm{H}_{11} \mathrm{~N}_{2} \mathrm{O}_{4}\right]^{+}: 295$ (65\%), $\left[\left(\mathrm{C}_{4} \mathrm{H}_{9}\right)_{3} \mathrm{Sn}\right]^{+}: 289$ (57\%), $\left[\left(\mathrm{C}_{4} \mathrm{H}_{9}\right)_{2} \mathrm{Sn}\right]^{+}: 232(36 \%),\left[\left(\mathrm{C}_{4} \mathrm{H}_{9}\right) \mathrm{Sn}\right]^{+}: 175(50 \%)$, $[\mathrm{Sn}]^{+}: 119(58 \%),\left[\mathrm{CH}_{3} \mathrm{CH}_{2} \mathrm{CH}_{2}\right]^{+}: 43(69 \%),\left[\mathrm{CH}_{3} \mathrm{CH}_{2}\right]^{+}$: $29(57 \%) ;\left[\mathrm{CH}_{3}\right]^{+}: 15(47 \%)$.
Triphenyltin(IV)-3-phthalimido-3(2-nitrophenyl) propanoate (11)

Yield 78\%; mp $135{ }^{\circ} \mathrm{C}$; solubility: acetone, DMF, DMSO, THF; anal. calcd. (\%): C 56.9, H 4.7, N 2.8, found: C 56.7, H 4.5, N 2.7; FTIR $v_{\max } / \mathrm{cm}^{-1} 1686 v(\mathrm{COO})_{\text {asym }}$, $1495 v(\mathrm{COO})_{\text {sym }}, \Delta v 191,1720 v(\mathrm{~N}-\mathrm{CO})_{\text {asym }}, 1690$ $v(\mathrm{~N}-\mathrm{CO})_{\text {sym }}, 1210 v(\mathrm{C}-\mathrm{N}), 543 v(\mathrm{Sn}-\mathrm{C}), 505 v(\mathrm{Sn}-\mathrm{O})$; ${ }^{1} \mathrm{H}$ NMR (400 MHz, DMSO) $\delta 1.31$ (2H, d, $\left.J 7.3 \mathrm{~Hz}, \mathrm{H} 2\right)$, 1.40 (1H, t, J 7.5 Hz, H3), 6.75 (1H, d, J 7.3 Hz, H6), 6.79 (1H, d, J 7.1 Hz, H7), $7.74(1 \mathrm{H}, \mathrm{d}, J 7.5 \mathrm{~Hz}, \mathrm{H} 10), 7.93$ $(1 \mathrm{H}, \mathrm{d}, J 7.1 \mathrm{~Hz}, \mathrm{H} 11), 7.74(1 \mathrm{H}, \mathrm{t}, J 7.0 \mathrm{~Hz}, \mathrm{Hb}), 7.65(1 \mathrm{H}$, m, Hc), $7.61(1 \mathrm{H}, \mathrm{t}, J 7.0 \mathrm{~Hz}, \mathrm{Hd}) ;{ }^{13} \mathrm{C}$ NMR $(100 \mathrm{MHz}$, DMSO) $\delta 176.52$ (C1), 43.50 (C2), 29.85 (C3), 121.45 (C4), 124.20 (C5), 127.35 (C6), 131.54 (C7), 163.91 (C8), 133.85 (C9), 135.15, (C10), 137.42 (C11), 138.65 (Ca), 140.24 (Cb), 141.58 (Cc), 143.25 (Cd); ${ }^{119} \mathrm{Sn}$ NMR $\left(\mathrm{CH}_{3}\right)_{4} \mathrm{Sn}$ : 96.84; EI-MS m/z, $\left[\left(\mathrm{C}_{6} \mathrm{H}_{5}\right)_{3} \mathrm{Sn}\left\{\mathrm{O}_{2} \mathrm{CC}_{16} \mathrm{H}_{11} \mathrm{~N}_{2} \mathrm{O}_{4}\right\}\right]^{+}$: 688 (42\%), $\left[\left(\mathrm{C}_{6} \mathrm{H}_{5}\right)_{2} \mathrm{Sn}\left\{\mathrm{O}_{2} \mathrm{CC}_{16} \mathrm{H}_{11} \mathrm{~N}_{2} \mathrm{O}_{4}\right\}\right]^{+}: 611$ (100\%), $\left[\left(\mathrm{C}_{6} \mathrm{H}_{5}\right)_{2} \mathrm{Sn}\left\{\mathrm{C}_{16} \mathrm{H}_{11} \mathrm{~N}_{2} \mathrm{O}_{4}\right\}\right]^{+}: 567(54 \%),\left[\left(\mathrm{C}_{6} \mathrm{H}_{5}\right)\right.$ $\left.\mathrm{Sn}\left\{\mathrm{C}_{16} \mathrm{H}_{11} \mathrm{~N}_{2} \mathrm{O}_{4}\right\}\right]^{+}: 490(28 \%),\left[\left(\mathrm{C}_{6} \mathrm{H}_{5}\right)_{3} \mathrm{Sn}\right]^{+}: 349(50 \%)$, $\left[\mathrm{C}_{16} \mathrm{H}_{11} \mathrm{~N}_{2} \mathrm{O}_{4}\right]^{+}: 295(61 \%),\left[\left(\mathrm{C}_{6} \mathrm{H}_{5}\right)_{2} \mathrm{Sn}\right]^{+}: 272(44 \%),\left[\left(\mathrm{C}_{6} \mathrm{H}_{5}\right)\right.$ $\mathrm{Sn}]^{+}: 195(50 \%),[\mathrm{Sn}]^{+}: 119(56 \%),\left[\mathrm{C}_{6} \mathrm{H}_{5}\right]^{+}: 77$ (65\%).

Tricyclohexyltin(IV)-3-phthalimido-3(2-nitrophenyl) propanoate (12)

Yield 79\%; mp $125{ }^{\circ} \mathrm{C}$; solubility: acetone, DMF, DMSO, THF; anal. calcd. (\%): C 54.9, H 7.9, N 2.7, found: C 54.7, H 7.8, N 2.6; FTIR $v_{\max } / \mathrm{cm}^{-1} 1650 v(\mathrm{COO})_{\text {asym }}$, $1495 v(\mathrm{COO})_{\mathrm{sym}}, \Delta v 155,1740 v(\mathrm{~N}-\mathrm{CO})_{\text {asym }}, 1646$ $v(\mathrm{~N}-\mathrm{CO})_{\text {sym }}, 1020 v(\mathrm{C}-\mathrm{N}), 560 v(\mathrm{Sn}-\mathrm{C}), 538 v(\mathrm{Sn}-\mathrm{O})$; ${ }^{1} \mathrm{H}$ NMR (400 MHz, DMSO) $\delta 1.29$ (2H, d, J 7.3 Hz, H2), $1.41(1 \mathrm{H}, \mathrm{t}, J 7.5 \mathrm{~Hz}, \mathrm{H} 3), 6.97$ (1H, d, J 7.3 Hz, H6), 7.69 $(1 \mathrm{H}, \mathrm{d}, J 7.1 \mathrm{~Hz}, \mathrm{H} 7), 7.83(1 \mathrm{H}, \mathrm{d}, J 7.5 \mathrm{~Hz}, \mathrm{H} 10), 7.89$ $(1 \mathrm{H}, \mathrm{d}, J 7.1 \mathrm{~Hz}, \mathrm{H} 11), 1.18(2 \mathrm{H}, \mathrm{t}, J 7.0 \mathrm{~Hz}, \mathrm{Ha}), 1.32$ $(2 \mathrm{H}, \mathrm{m}, \mathrm{Hb}) 1.65(1 \mathrm{H}, \mathrm{m}, \mathrm{Hc}), 1.79(1 \mathrm{H}, \mathrm{m}, \mathrm{Hd}) ;{ }^{13} \mathrm{C} \mathrm{NMR}$ (100 MHz, DMSO) $\delta 170.45$ (C1), 4.5.50 (C2), 30.15 (C3), 125.50 (C4), 129.15 (C5), 131.40 (C6), 133.25 (C7), 164.51 (C8), 134.25 (C9), 137.75 (C10), 138.65 (C11), 28.19 (Ca), $27.40(\mathrm{Cb}), 26.54(\mathrm{Cc}), 23.95(\mathrm{Cd}) ;{ }^{119} \mathrm{Sn} \mathrm{NMR}\left(\mathrm{CH}_{3}\right)_{4} \mathrm{Sn}$ : 9.72; EI-MS $m / z,\left[\left(\mathrm{C}_{6} \mathrm{H}_{11}\right)_{3} \mathrm{Sn}\left\{\mathrm{O}_{2} \mathrm{CC}_{16} \mathrm{H}_{11} \mathrm{~N}_{2} \mathrm{O}_{4}\right\}\right]^{+}: 706$ (45\%), $\left[\left(\mathrm{C}_{6} \mathrm{H}_{11}\right)_{2} \mathrm{Sn}\left\{\mathrm{O}_{2} \mathrm{CC}_{16} \mathrm{H}_{11} \mathrm{~N}_{2} \mathrm{O}_{4}\right\}\right]^{+}: 623$ (76\%), $\left[\left(\mathrm{C}_{6} \mathrm{H}_{11}\right)_{2} \mathrm{Sn}\left\{\mathrm{C}_{16} \mathrm{H}_{11} \mathrm{~N}_{2} \mathrm{O}_{4}\right\}\right]^{+}: 579(58 \%),\left[\left(\mathrm{C}_{6} \mathrm{H}_{11}\right)\right.$ $\left.\operatorname{Sn}\left\{\mathrm{C}_{16} \mathrm{H}_{11} \mathrm{~N}_{2} \mathrm{O}_{4}\right\}\right]^{+}: 496(100 \%),\left[\left(\mathrm{C}_{6} \mathrm{H}_{11}\right)_{3} \mathrm{Sn}\right]^{+}: 367(43 \%)$, $\left[\mathrm{C}_{16} \mathrm{H}_{11} \mathrm{~N}_{2} \mathrm{O}_{4}\right]^{+}: 295(55 \%),\left[\left(\mathrm{C}_{6} \mathrm{H}_{11}\right)_{2} \mathrm{Sn}\right]^{+}: 284(57 \%)$, $\left[\left(\mathrm{C}_{6} \mathrm{H}_{11}\right) \mathrm{Sn}\right]^{+}: 195(50 \%),[\mathrm{Sn}]^{+}: 119(56 \%),\left[\mathrm{C}_{6} \mathrm{H}_{11}\right]^{+}: 83$ $(41 \%)$.

\section{Bioactivity studies}

Antibacterial activity

The $\beta$-amino acid derivatives P2HPA, P2NPA and 
organotin(IV) complexes (1-12) were tested by disc diffusion method against four standard bacterial strains, i.e., $S$. aureus, B. subtilis, E. coli and P. aeruginosa. Before utilizing, all the glassware were sterilized at $150^{\circ} \mathrm{C}$ for $20 \mathrm{~min}$. For the antibacterial testing, the microbial specimens as swabs of pus, blood, urine, sputum, etc., were collected, isolated and accumulated from different wards of Nishter Hospital Multan. MacConkey agar (10.0 g) and CLED (cystine-lactose-electrolyte-deficient) mediums $(10.0 \mathrm{~g})$ in $250 \mathrm{~mL}$ of distilled water were autoclaved and proceeded further to prepare Petri plates. The solutions of the ligands and complexes (1-12) of $500 \mathrm{ppm}$ were formulated in DMSO. The composed discs after drenching in test solutions were dried and autoclaved. All the developed Petri plates were subjected to incubation at $37^{\circ} \mathrm{C}$ for $24 \mathrm{~h}$. Ciprofloxacin was taken as a standard and DMSO as a negative control. This procedure was repeated in triplicate. ${ }^{34}$

\section{Molecular docking}

\section{Receptor and ligand preparation}

The crystal structure of sortase A in complex with LPETG was downloaded from Protein Data Bank with (PDB ID 1T2W). ${ }^{35}$ The receptor was prepared by removing the ligand LPETG and water molecules. Vina configuration file was generated by using the AutoDock Tools software ${ }^{36}$ (version 4.2) with polar hydrogen and Gasteiger charges were added to finally obtain the file in PDBQT format. The location of final size space dimension of the grid was used $x=18 \AA, y=18 \AA$ and $z=18 \AA$ and center -33.604 , -17.684 and 7.115 for $x, y$ and $z$ coordinate respectively. Ligands were drawn and energy was minimized by using MarvinSketch software, ${ }^{37}$ saved as mol 2 format, the Gastiger charges were added by AutoDock Vina ${ }^{37}$ tools and change the files into PDBQT format. ${ }^{37}$ Discovery Studio Client 2019 was used for 2D and 3D molecular interaction. ${ }^{38}$

\section{Antioxidant activity by DPPH method (free radical scavenging)}

The ligands P2HPA, P2NPA and organotin(IV) complexes (1-12) were subjected to DPPH (1,1-diphenyl2-picrylhydrazyl radical) method for antioxidant activity. In this procedure, about $0.5 \mathrm{mg} \mathrm{mL}^{-1}$ of each compound in corresponding solvents were poured at an equal volume $(10 \mu \mathrm{L})$ to $90 \mu \mathrm{L}$ of $100 \mu \mathrm{M}$ methanolic DPPH in a total volume of $100 \mu \mathrm{L}$ in dried well plates and incubated at $37^{\circ} \mathrm{C}$ for $30 \mathrm{~min}$. The experiment was repeated in triplicate. The Synergy HT BioTek ${ }^{\circledR}$ USA microplate reader measured the absorbance at $517 \mathrm{~nm}$. The standard antioxidant used was quercetin. The solution of methanolic DPPH served as a negative control. The decrease in absorbance is the indication of increased radical scavenging activity and was measured by the equation $1 .{ }^{39}$

Scavenging activity $(\%)=[(\mathrm{Ac}-\mathrm{As}) / \mathrm{Ac}] \times 100$

where, As: absorbance of sample and Ac: absorbance of negative control.

\section{Antidiabetic activity}

In this study, 24 adult male healthy rabbits weighing about 1 to $2 \mathrm{~kg}$ were taken and categorized into eight groups (GI-GVIII) with 3 rabbits in each group. One group served as a control group and the other seven are experimental groups. Rabbits were made diabetic by injecting alloxan at $150 \mathrm{mg} \mathrm{kg}^{-1}$ of body weight. Before injecting alloxan, the normal blood glucose level of all rabbits was determined and noted. After $24 \mathrm{~h}$ of alloxan injection, the rabbits were considered as diabetic because the blood glucose levels of all the rabbits were found between 220 to $350 \mathrm{mg} \mathrm{dL}^{-1}$ and were employed to further study. ${ }^{40}$ The test solutions of all the compounds were prepared by dissolving different concentrations, i.e., $0.5,1.0,1.5 \mathrm{mg}$ of the compounds in DMSO. Metformin acted as a standard drug and was subjected to the control group. After administration of the test solutions to all the diabetic rabbits, the blood sample was collected after every 3, 6, 9, 12 and $24 \mathrm{~h}$ and blood glucose level was estimated by glucometer (SD Biosensor, INC.) by the glucose oxidase method. ${ }^{41}$ The process number of ethics committee associated with article is No. ICS/D-297/20.

\section{Results and Discussion}

\section{FTIR study}

The IR spectra of the ligands P2HPA and P2NPA indicated the absence of bands at $3500-3100 \mathrm{~cm}^{-1}$ due to amino group $\left(\mathrm{NH}_{2}\right)$. A new prominent peak of $v(\mathrm{C}-\mathrm{N})$ appeared at $1270-1200 \mathrm{~cm}^{-1}$ due to the imide linkage and this was the indication of the entrance of $\mathrm{N}$ in the anhydride ring. The $v(\mathrm{OH})$ peak of carboxylic acid was observed at $3300-2500 \mathrm{~cm}^{-1}$ and $v(\mathrm{C}-\mathrm{H})$ at $3200-2961 \mathrm{~cm}^{-1}$. The stretching frequencies of the carboxylic group emerged as a strong band at about $1750-1620 \mathrm{~cm}^{-1}$ due to $v(\mathrm{COO})_{\text {asym }}$ and at $1740-1610 \mathrm{~cm}^{-1}$ due to $v(\mathrm{COO})_{\mathrm{sym}}$ in ligands. The characteristic $v(\mathrm{~N}-\mathrm{C}=\mathrm{O})$ peaks of imide linkage were observed at 1790-1760 and 1780-1750 $\mathrm{cm}^{-1}$. The peaks at $1600-1500 \mathrm{~cm}^{-1}$ were assigned to the aromatic 
benzene ring (Figures S1-S2, Supplementary Information (SI) section) ${ }^{22,23}$ The important $v(\mathrm{COO})_{\text {asym }}, v(\mathrm{COO})_{\text {sym }}$, $v(\mathrm{Sn}-\mathrm{C})$ and $v(\mathrm{Sn}-\mathrm{O})$ peaks were observed in the spectra of complexes at their proper positions. ${ }^{42}$ The vanishing of the broad peak $v(\mathrm{OH})$ carboxylic acid in the range of $3300-2500 \mathrm{~cm}^{-1}$ and emergence of $v(\mathrm{Sn}-\mathrm{O})$ at $538-502 \mathrm{~cm}^{-1}$ suggested the formation of complexes, deprotonation and coordination of the carboxylate group to tin(IV) metal. ${ }^{43-45}$ Moreover, it was also observed that the carboxylate group $v(\mathrm{COO})$ value in metal complexes had been shifted to a lower frequency as compared to that of free ligands which indicated the participation of the $v(\mathrm{COO})$ group in coordination (Figures S3-S14, SI section). ${ }^{46}$ The carboxylate group coordination mode of a ligand with metal can be determined by the difference in asymmetric $v(\mathrm{COO})_{\text {asym }}$ and symmetric $v(\mathrm{COO})_{\text {sym }}$ frequencies, i.e., $\Delta v(\mathrm{COO}){ }^{47-49}$ The $\Delta v$ values $>200 \mathrm{~cm}^{-1}$ favors monodentate and $<200 \mathrm{~cm}^{-1}$ suggests bidentate coordination while chelation observes if the value is $<150 \mathrm{~cm}^{-1} \cdot{ }^{50,51} \mathrm{In}$ monomeric complexes ( $\mathbf{1}$, $\mathbf{3}, \mathbf{7}, \mathbf{9}), v(\mathrm{COO})_{\text {asym }}$ and $v(\mathrm{COO})_{\text {sym }}$ intense and sharp bands appeared in the range of $1650-1630$ and $1472-1440 \mathrm{~cm}^{-1}$, respectively, while the $\Delta v(\mathrm{COO})$ values were less than $200 \mathrm{~cm}^{-1}$. This exhibited bidentate coordination of ligands with trans-octahedral geometry (Figure 1a). ${ }^{52}$ In dimeric complexes $(2,8)$ intense bands at 502 and $510 \mathrm{~cm}^{-1}$ proposed ring dimeric network of $\mathrm{Sn}-\mathrm{O}-\mathrm{Sn}-\mathrm{O}$ with endo and exo tin(IV) atoms with hexa-coordinated geometry and the $v(\mathrm{COO})_{\text {asym }}$ and $v(\mathrm{COO})_{\text {sym }}$ frequencies were in the range of 1628-1608 and $1475-1455 \mathrm{~cm}^{-1}$, respectively (Figure 1b). ${ }^{31,52}$ The distinctive $v(\mathrm{COO})_{\text {asym }}$ and $v(\mathrm{COO})_{\text {sym }}$ frequencies of triorganotin(IV) compounds (4-6, 10-12) appeared in the range of 1686-1610 and $1510-1451 \mathrm{~cm}^{-1}$, respectively, and $\Delta v(\mathrm{COO})$ values suggested that $\mathrm{SnR}_{3}$ groups have bidentate coordination with trigonal bipyramidal geometry in solid-state (Figure 1c). ${ }^{52-54}$ While the $v(\mathrm{Sn}-\mathrm{C})$ stretching vibrations were observed at about $560-512 \mathrm{~cm}^{-1}$ for cyclohexyl, butyl and phenyl groups which are in good agreement with the literature. ${ }^{55,56}$

\section{${ }^{1} \mathrm{H}$ NMR study}

The ${ }^{1} \mathrm{H}$ NMR study of the ligands P2HPA, P2NPA and metal complexes (1-12) successfully verified their synthesis. The prominent resonance peaks in the ${ }^{1} \mathrm{H}$ NMR spectra of ligands indicated the presence of $-\mathrm{OH}$ proton at $12.00-10.00 \mathrm{ppm}$ while the absence of this signal in the spectra of complexes revealed that during complexation deprotonation of the carboxylic group have taken place (Figures 2a, S15-S16, SI section). ${ }^{44,45}$ Moreover, the proton signals of $-\mathrm{NH}_{2}$ group at the range of 0.5-5.0 ppm were also absent in the spectra of ligands. The multiplet signals of aromatic protons at 7.49-7.24 ppm were observed both in the ligands and also in complexes. The $-\mathrm{CH}_{2}$ and $-\mathrm{CH}$ chemical shifts were found at 1.31-1.20 and 1.45-1.34 ppm in the spectra of ligands and complexes.

The butyl group attached to tin(IV) gave a multiplet signal due to $-\mathrm{CH}_{2}-\mathrm{CH}_{2}-\mathrm{CH}_{2}-$ protons at 2.01-1.14 ppm and a triplet signal at the $0.95-0.80 \mathrm{ppm}$ region due to terminal $-\mathrm{CH}_{3}$ (Figures $2 \mathrm{~b}, \mathrm{~S} 17-\mathrm{S} 28$, SI section). The proton signals of phenyl were at 7.74-7.15 ppm region and cyclohexyl were found at 1.81-1.14 ppm region, respectively. ${ }^{56-58}$

The ${ }^{1} \mathrm{H}$ NMR data confirmed the synthesis of monomer complexes $(\mathbf{1}, \mathbf{3}, \mathbf{7}, \mathbf{9})$ with octahedral geometry (Figure 1a). ${ }^{59}$ In case of dimer complexes $(\mathbf{2}, \mathbf{8})$ it was presumed that prominent butyl proton signals of endo and exo tin(IV) centers were absent, which indicated the identical environment of exo and endo tin(IV) and thus confirmed a unique view having hexa coordinated geometry with the neighboring tin(IV) center of one unit being linked by other unit through an oxygen atom of the carboxylate group (Figure 1b). ${ }^{31,60,61}$ The triorganotin(IV) complexes (4-6, 10-12) verified the tetrahedral structure and exhibited that oxygen of the carboxylate group was coordinated to tin(IV) in this arrangement (Figure 1d)..$^{60,61}$

\section{${ }^{13} \mathrm{C}$ NMR study}

The ${ }^{13} \mathrm{C}$ NMR spectral data represented the characteristic peaks of organotin(IV) complexes (1-12) and ligands. The signals due to the carboxylate group carbon in the ligands appeared at the range of $169.38-167.83 \mathrm{ppm}$. The $-\mathrm{CH}$ and $-\mathrm{CH}_{2}$ carbon signals were observed at 36.75-30.09 and 49.27-45.69 ppm, respectively. The aromatic carbons of the benzene ring gave signals at $133.27-116.50 \mathrm{ppm}$ while the imide group $(\mathrm{N}-\mathrm{C}=\mathrm{O})$ carbon signals were observed in the range of 165.33-162.84 ppm, thus confirming their obtainment (Figures 2c, S29-S30, SI section). ${ }^{44,45}$

However, in complexes the carboxylate group chemical shift values appeared downfield at the range of 177.65-170.25 ppm than the values observed in ligands (Figures 2d, S31-S42, SI section). The chemical shift values of the carboxylate carbon suggested the participation of carboxylate oxygen during complexation. ${ }^{32,52}$ The chemical shift values of butyl, phenyl and cyclohexyl groups $(\mathrm{Ca}, \mathrm{Cb}$, $\mathrm{Cc}, \mathrm{Cd}$ ) appeared at 29.65-12.12, 143.39-137.61 and 28.89$12.55 \mathrm{ppm}$, respectively, as reported in literature. ${ }^{32,52,60}$

${ }^{119}$ Sn NMR study

The ${ }^{119}$ Sn NMR chemical shift values marked the coordination number and predicted the geometries around 
<smiles>[R]O[N+](=O)[O-]</smiles>

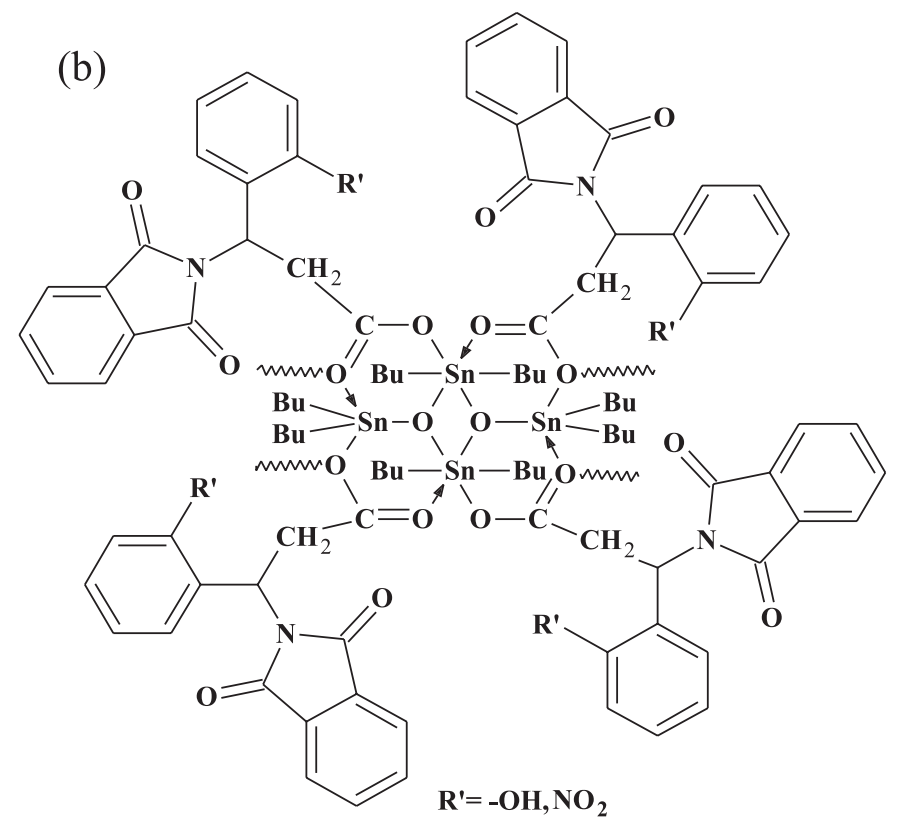

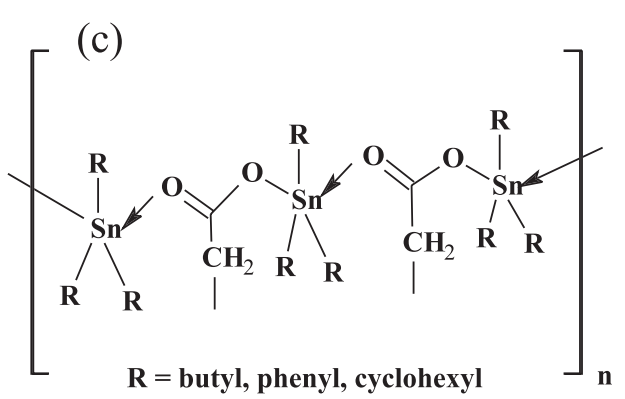<smiles>[R]O[N+]([R])([R])OC(=O)CC(c1ccccc1[R])N1C(=O)c2ccccc2C1=O</smiles>

Figure 1. (a) A monomer unit having octahedral geometry, (b) a dimer unit, (c) trigonal bipyramidal geometry, (d) tetrahedral geometry.

tin(IV) atoms. ${ }^{32}$ In case of dibutyltin(IV) monomer complexes $(\mathbf{1}, \mathbf{3}, \mathbf{7}, \mathbf{9}){ }^{119} \mathrm{Sn}$ chemical shift values appeared at $197.54-185.58 \mathrm{ppm}$, thus confirmed a trans octahedral geometry (Figure 1a). ${ }^{62}$ In dimmers $(\mathbf{2}, \mathbf{8})$ the endo and exo cyclic status of tin(IV) was confirmed by two equal intensity peaks at 215.25 and $216.24 \mathrm{ppm}$, respectively, and substantiated a hexa-coordinated geometry as given in (Figure 1b) ${ }^{63}$ While in triorganotin(IV) complexes the chemical shift values at 135.79-123.71, 99.28-96.84 and 9.72-9.15 ppm for butyl, phenyl and cyclohexyl (4-6, 10-12) supported a tetrahedral environment (Figure 1d). ${ }^{64}$
Mass spectrometry study

In ligands P2HPA and P2NPA molecular ion peaks at $\mathrm{m} / \mathrm{z} 311$ and 340 represented their actual masses and confirmed their synthesis (Schemes 3 and 4 and Figures S43-S44, SI section). ${ }^{31,32}$ In the fragmentation of the triorganotin(IV) derivatives (4-6, 10-12) the ligand moiety lost was the cause of major fragmentation. The $\mathrm{R}$ groups (butyl, phenyl, cyclohexyl) during fragmentation were lost successively until the $\mathrm{Sn}^{4+}$ ion resulted. In another possible route, the $\mathrm{R}$ groups were lost in the first step and the next 

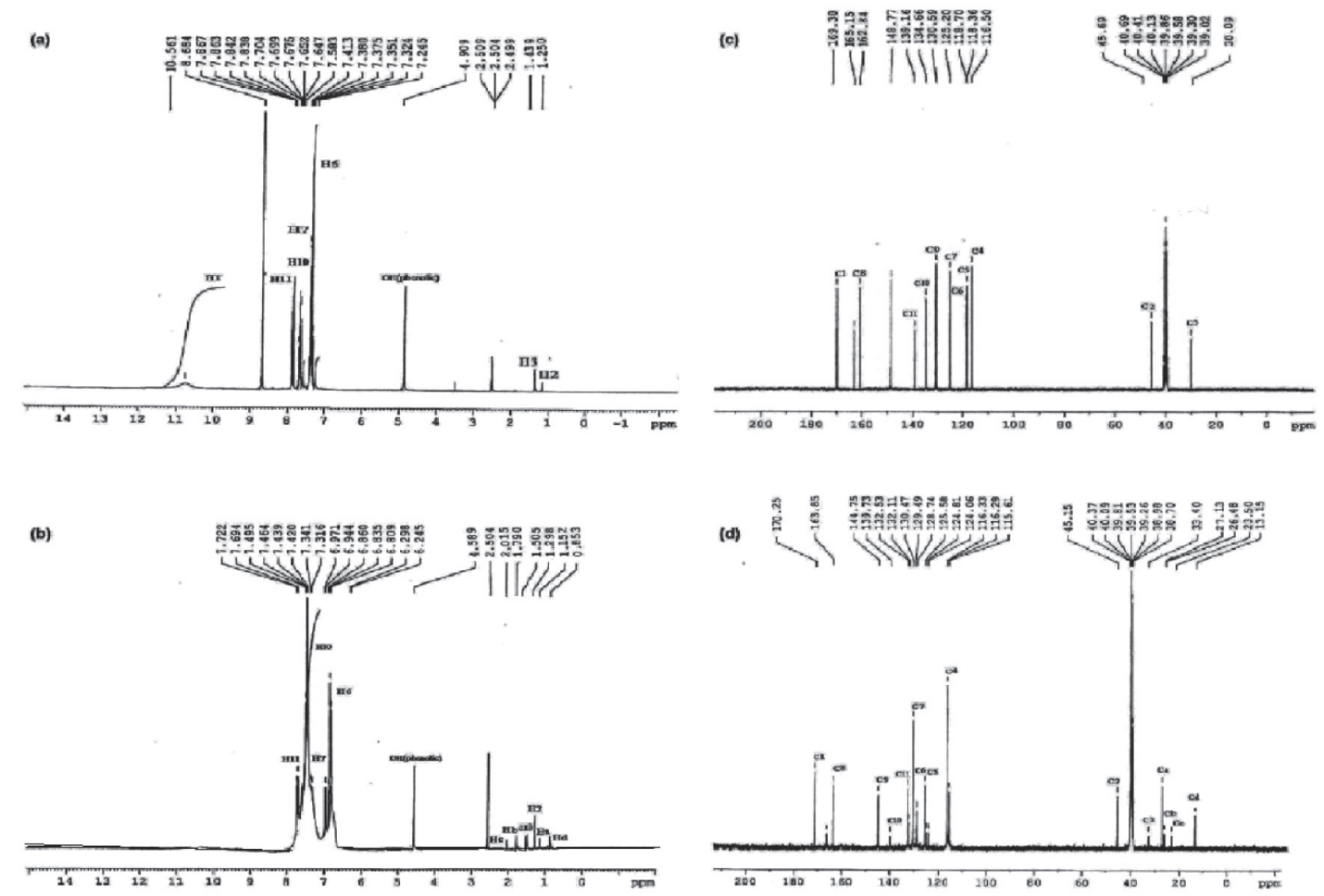

Figure 2. ${ }^{1} \mathrm{H}$ NMR spectrum (400 MHz, DMSO) of the ligand P2HPA and its monomer complex (a, b), ${ }^{13} \mathrm{C}$ NMR spectrum (100 MHz, DMSO) of the ligand P2HPA and its monomer complex (c, d).

step would be the elimination of one molecule of $\mathrm{CO}_{2}$. Further, the remaining substituents were lost in successive steps on almost similar patterns (Scheme 5). ${ }^{31,65}$ In the diorganotin(IV) derivatives $(\mathbf{1 - 3}, \mathbf{7 - 9})$ the major cause of

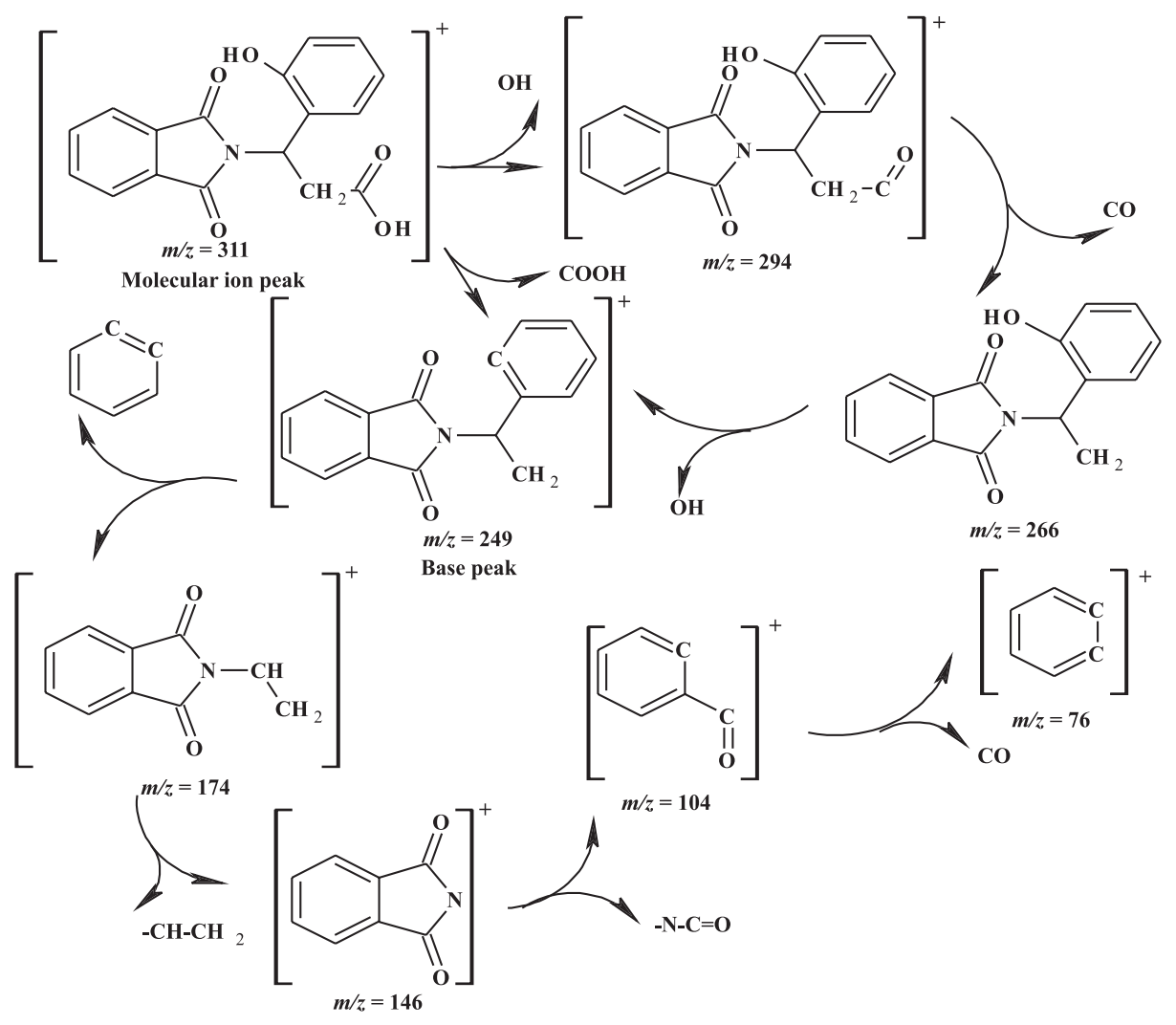

Scheme 3. Fragmentation pattern of the ligand P2HPA. 


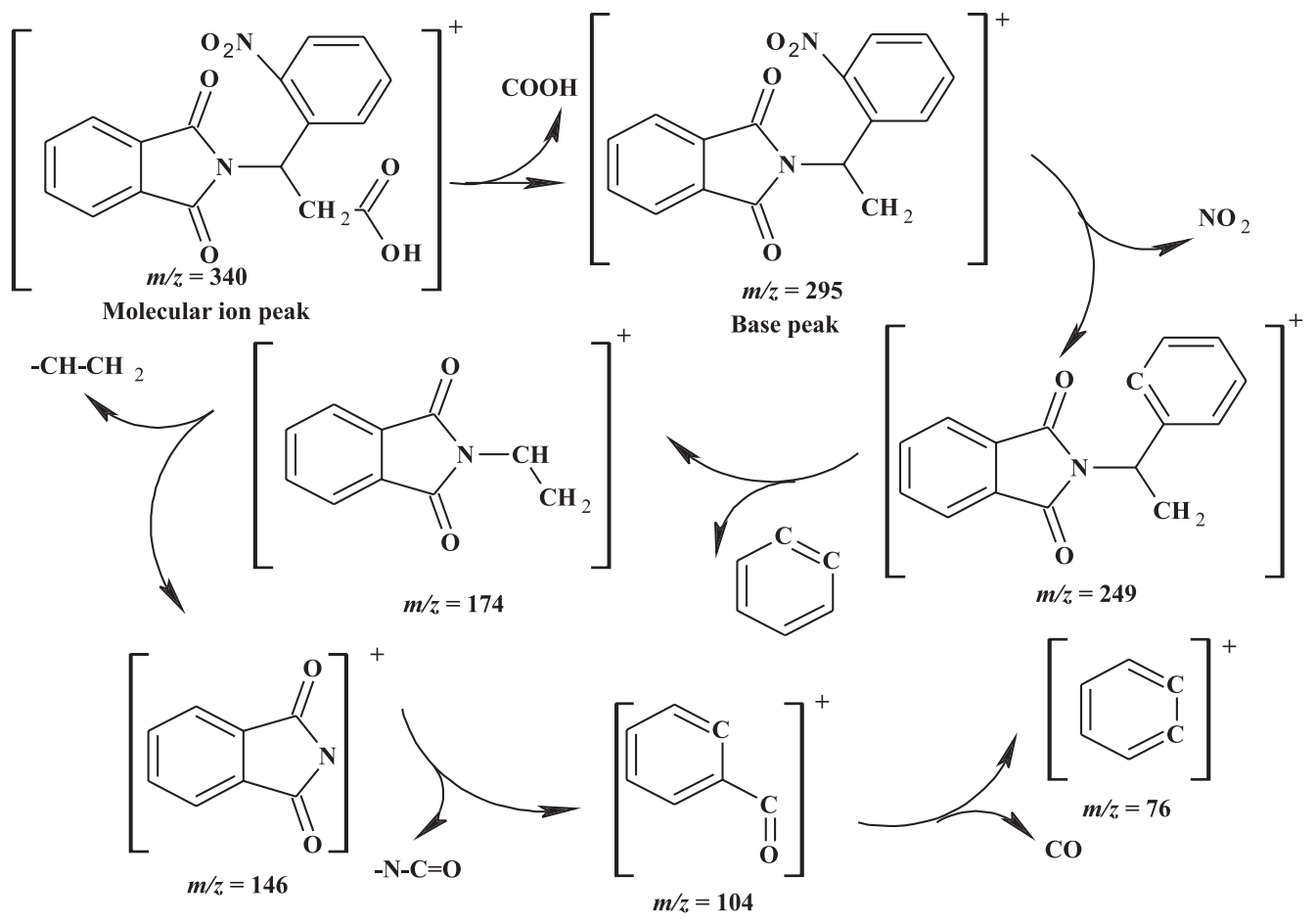

Scheme 4. Fragmentation pattern of the ligand P2NPA.

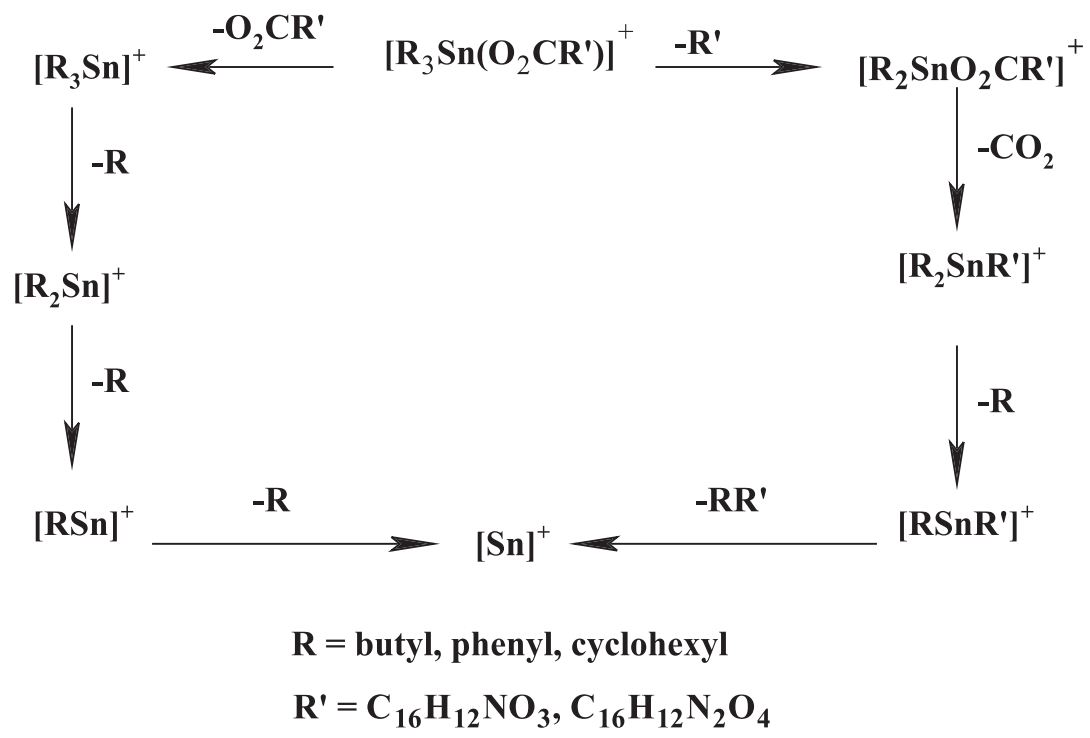

Scheme 5. Fragmentation pattern of triorganotin(IV) complexes.

fragmentation was the elimination of the ligand and $\mathrm{CO}_{2}$ molecule. The R groups (butyl, phenyl, cyclohexyl) and the existing complex counterparts were lost successively until the $\mathrm{Sn}^{4+}$ ion was obtained. An alternative route followed the loss of $\mathrm{R}$ group in first step and then two molecules of $\mathrm{CO}_{2}$ and remaining $\mathrm{R}$ groups. The third rout suggested the loss of $\mathrm{CO}_{2}$ molecule first and then the remaining substituents including the ligand and R (butyl, phenyl, cyclohexyl) groups (Scheme 6). ${ }^{33,66}$ The spectra of all the complexes are presented in Figures S45-S56 (SI section).

\section{Biological studies}

\section{Antibacterial bioassay}

Antibacterial studies of the synthesized ligands P2HPA, P2NPA and complexes 1-12 were performed using four strains of bacteria (S. aureus, B. subtilis, E. coli and $P$. aeruginosa) as shown in Table 1 and ciprofloxacin was used as a reference drug. The studies suggested that the $\beta$-amino acid derivatives were significantly active biological compounds, but the antibacterial activity of their 


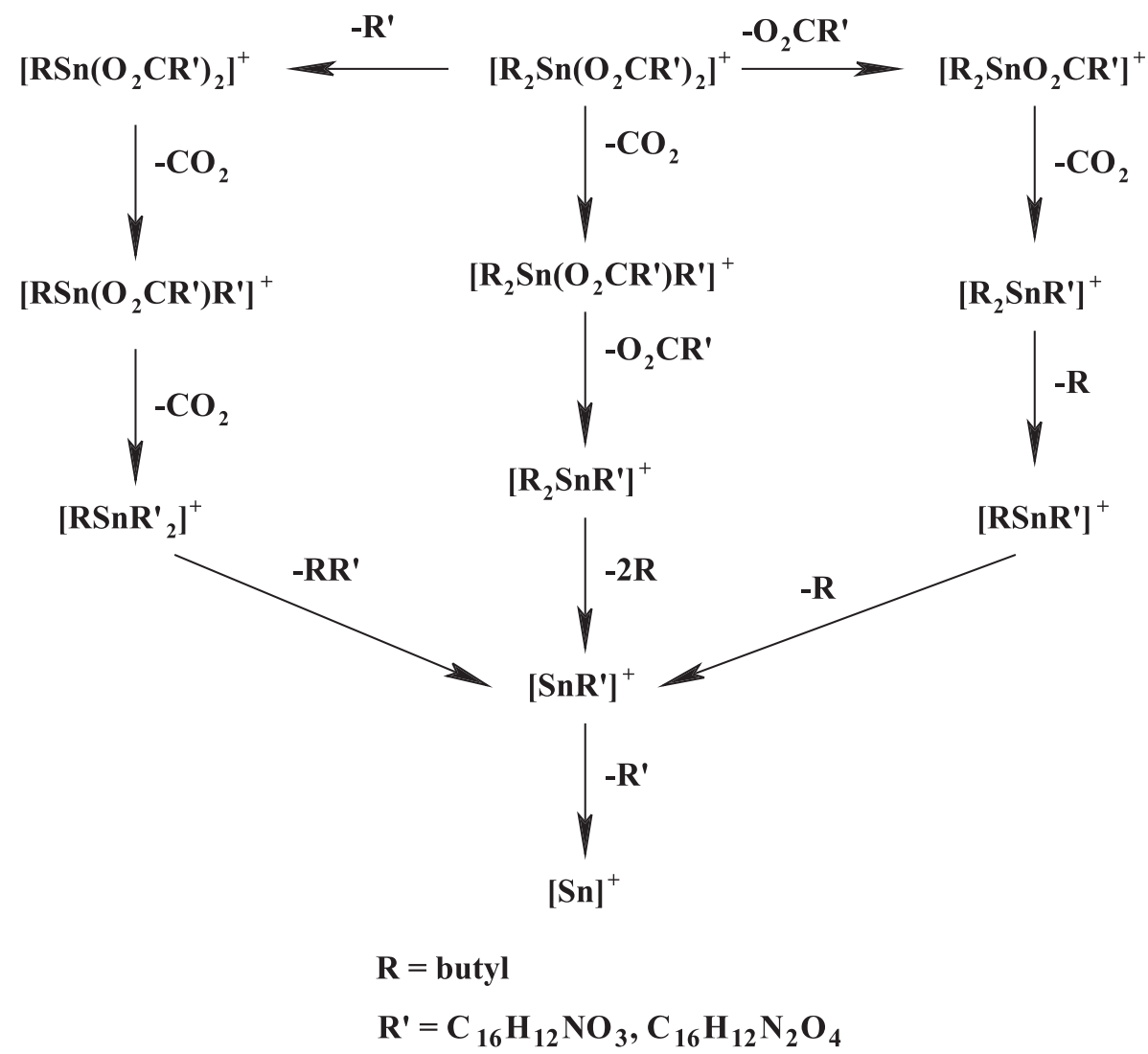

Scheme 6. Fragmentation pattern of diorganotin(IV) complexes.

respective metal complexes was found remarkably higher as compared to the ligands (Figure 3). ${ }^{67}$ The chelation and the addition of a substrate were the two factors behind the enhanced activity of the complexes. ${ }^{68}$

The triorganotin(IV) complexes (4-6, 10-12) demonstrated greater activity, having values in the range of 32-20 $\mathrm{mm}$, than diorganotin(IV) complexes (1-3, 7-9) that have values in the range of $25-10 \mathrm{~mm}$ at $500 \mathrm{ppm}$ dose. It was probably because of the coordination of carboxylate oxygen with substrate, thus resulting in tetrahedral geometry of tin(IV) atom rather than trigonal bipyramid. ${ }^{69}$ From this study, the following trend of bacterial inhibition for the organotin(IV) compounds was concluded: triorganotins (alkyl) $>$ triorganotins $($ aryl $)>$ dimer $>$ monomer $>$ ligand. However, differences were observed between Gram-positive and Gram-negative strains. The compounds were more active for positive strains. The bacterial strains increasing inhibition order was: E. coli $>$ P. aeruginosa $>$ S. aureus $>$ B. subtilis.

\section{Molecular docking studies}

The molecular docking studies were performed to find the best predicted binding affinities. To understand the established interaction and antimicrobial effectiveness of
Table 1. Antibacterial bioassay

\begin{tabular}{|c|c|c|c|c|}
\hline \multirow{3}{*}{$\begin{array}{l}\text { Ligand/ } \\
\text { complex } \\
(500 \mathrm{ppm})\end{array}$} & \multicolumn{4}{|c|}{ Inhibition $^{\mathrm{a}} / \mathrm{mm}$} \\
\hline & \multicolumn{2}{|c|}{ Gram-negative } & \multicolumn{2}{|c|}{ Gram-positive } \\
\hline & E. coli & $\overline{P .}$ aeruginosa & S. aureus & B. subtilis \\
\hline 1 & $18 \pm 0.2$ & $15 \pm 0.3$ & $23 \pm 0.2$ & $20 \pm 0.7$ \\
\hline 2 & $20 \pm 0.7$ & $18 \pm 0.4$ & $18 \pm 0.6$ & $25 \pm 0.5$ \\
\hline 3 & $12 \pm 0.1$ & $10 \pm 0.3$ & $15 \pm 0.1$ & $13 \pm 0.6$ \\
\hline 4 & $20 \pm 0.1$ & $18 \pm 0.6$ & $21 \pm 0.5$ & $30 \pm 0.1$ \\
\hline 5 & $28 \pm 0.3$ & $23 \pm 0.4$ & $29 \pm 0.4$ & $25 \pm 0.6$ \\
\hline 6 & $25 \pm 0.7$ & $22 \pm 0.4$ & $30 \pm 0.3$ & $26 \pm 0.8$ \\
\hline 7 & $24 \pm 0.6$ & $20 \pm 0.1$ & $19 \pm 0.1$ & $17 \pm 0.1$ \\
\hline 8 & $20 \pm 0.5$ & $18 \pm 0.1$ & $20 \pm 0.5$ & $18 \pm 0.5$ \\
\hline 9 & $24 \pm 0.9$ & $19 \pm 0.5$ & $20 \pm 0.1$ & $18 \pm 0.4$ \\
\hline 10 & $32 \pm 0.1$ & $28 \pm 0.3$ & $30 \pm 0.2$ & $27 \pm 0.2$ \\
\hline 11 & $28 \pm 0.1$ & $23 \pm 0.3$ & $26 \pm 0.3$ & $20 \pm 0.5$ \\
\hline 12 & $26 \pm 0.8$ & $22 \pm 0.1$ & $32 \pm 0.1$ & $26 \pm 0.8$ \\
\hline P2HPA & $14 \pm 0.6$ & $12 \pm 0.9$ & $22 \pm 0.6$ & $17 \pm 0.1$ \\
\hline P2NPA & $16 \pm 0.4$ & $15 \pm 0.6$ & $18 \pm 0.5$ & $16 \pm 0.4$ \\
\hline Standard ${ }^{\mathrm{b}}$ & $25 \pm 0.7$ & $28 \pm 0.3$ & $31 \pm 0.3$ & $30 \pm 0.1$ \\
\hline $\mathrm{DMSO}^{\mathrm{c}}$ & - & - & - & - \\
\hline
\end{tabular}

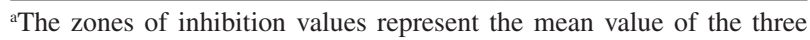

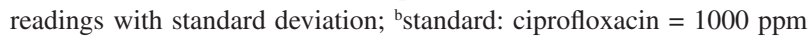
dose; 'DMSO: negative control. -: no activity. 


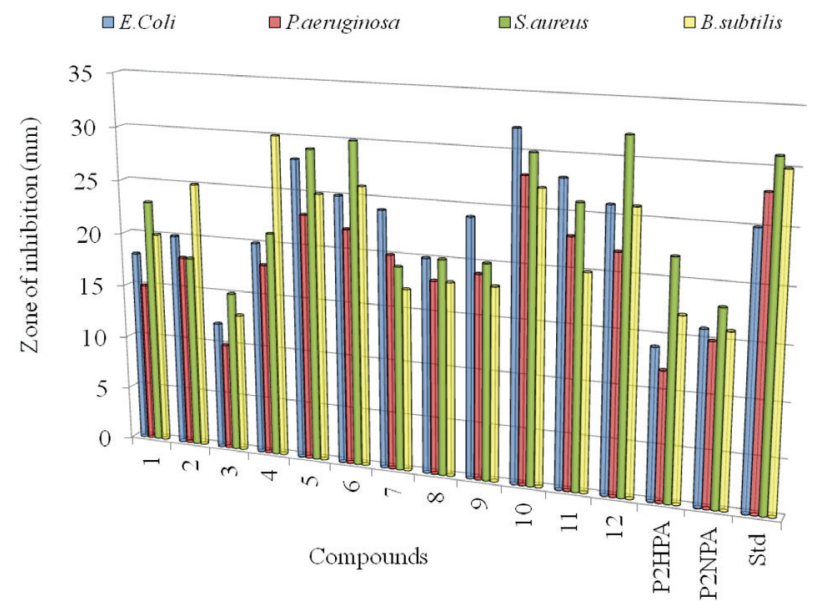

Figure 3. Antibacterial bioassay showing zone of inhibition with four bacterial strains.

proposed molecules P2HPA and P2NPA with important amino acid residues on the catalytic pocket of methicillinresistant $S$. aureus (MRSA) was studied. ${ }^{35}$ The enzyme selected for docking studies was retrieved from RSCB Protein Data Bank (PDB code 1T2W) with resolution
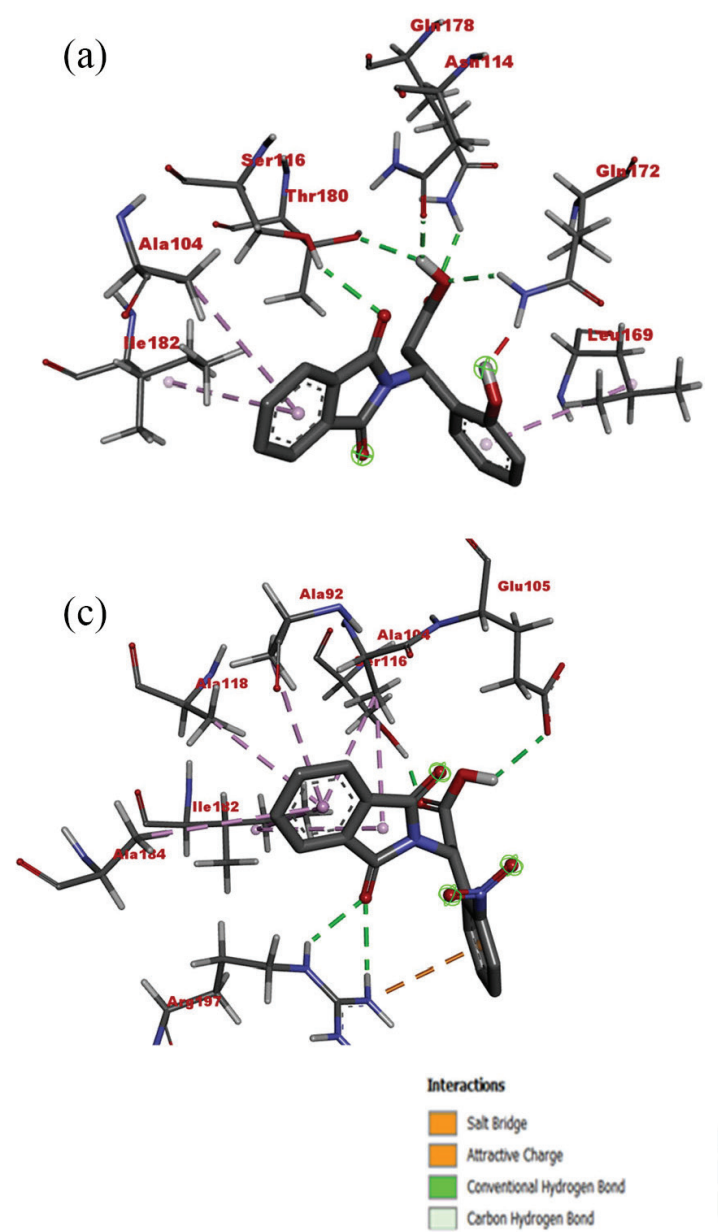

Figure 4. Representing, 3D interaction and 2D interaction of P2HPA (a, b) and P2NPA (c, d) ligands, respectively, on the catalytic pocket of (ID 1T2W) enzyme.
$1.80 \AA$ A. The size of the grid was configured keeping in view the size of ligands and important residues of the enzyme, i.e., Ala92, Ala104, Glu105, Ala118, Pro163, Leu169, Gln172, Thr180, Ile182, Trp194, and Ile199. ${ }^{36}$

Both ligand P2HPA and P2NPA showed $-6.5 \mathrm{~kJ} \mathrm{~mol}^{-1}$ binding affinities values but different poses of interaction on the catalytic pocket of the enzyme. The ligand P2HPA was found forming the different hydrogen bonds, $\pi$-alkyl interactions, and hydrophobic interactions with side chain residues of catalytic pocket. Three hydrogen bond interactions were formed by hydroxyl $(-\mathrm{OH})$ of propanoic acid with Asn114, Gln172 and The180, while the oxygen atom of the carbonyl group of propanoic acid was found in hydrogen bond interaction with amino acid residue Gln178. Moreover, the oxygen atom of the carbonyl group of phthaloyl moiety was found in hydrogen bond interaction with Ser116. In addition, the phenyl rings of ligand P2HPA were found in $\pi$-alkyl interactions with Ala104, Leu169 and Ile182. The amino acid residues Arg197, Val168, Pro163 and Asp170 were found forming the hydrophobic interactions with ligand P2HPA (Figures $4 \mathrm{a}$ and $4 \mathrm{~b}$ ). ${ }^{37}$

(b)

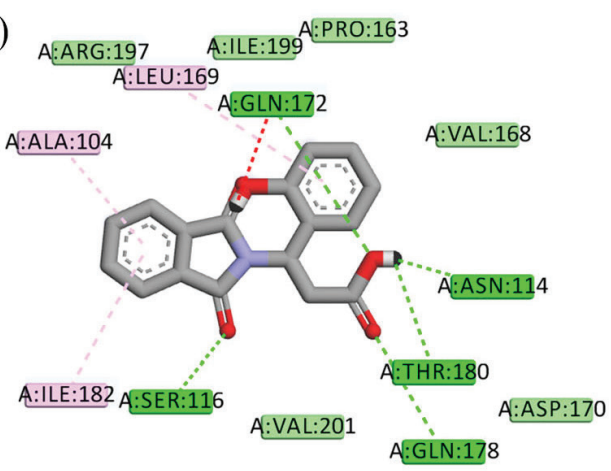

(d)
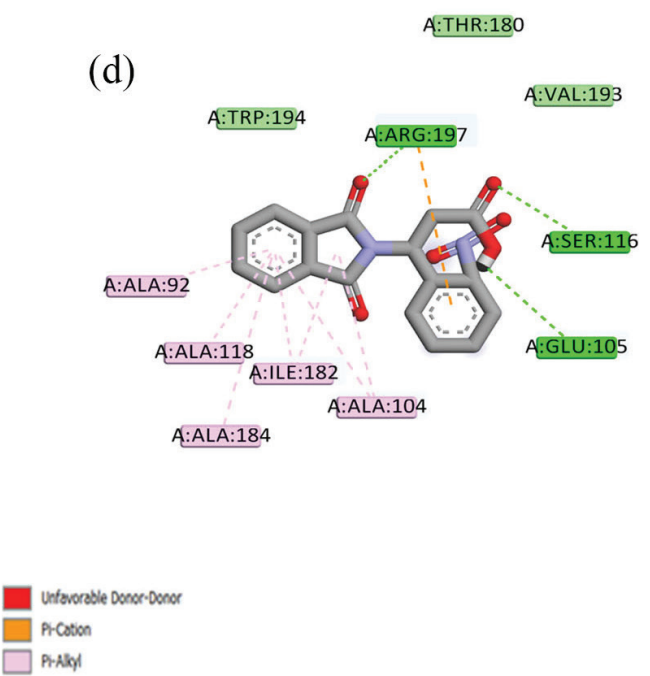
The ligand P2NPA was found forming the three hydrogen bond interactions, first between the oxygen atom of the carbonyl group of phthaloyl moiety and Arg197 residue, second between the oxygen atom of the carbonyl group of propanoic acid and Ser116, third between the hydrogen atom of hydroxyl group propanoic acid and Glu105 as hydrogen bond donor. The rest of amino acid residues Ala92, Ala118, Ala184, and Ile1812 showed the weak $\pi$-alkyl interactions with phthaloyl phenyl ring, while the amino acid residues Trp194, Thr180 and Val193 displayed the hydrophobic interactions (Figures $4 \mathrm{c}$ and 4d). ${ }^{38}$

Concluding the molecular docking studies, it can be summarized that the hydroxyl $(-\mathrm{OH})$ substituent on the phenyl ring of propanoic acid was more favorable compared to $-\mathrm{NO}_{2}$ substituent at same the position which may be due to more electron drawing ability and steric effect of $-\mathrm{NO}_{2}$ group. More interestingly, the ligand P2HPA which showed the excellent predicted binding interaction in the catalytic pocket also showed the high-value inhibition $22 \%$ compared to P2NPA, which showed only $18 \%$ inhibition for methicillin-resistant $S$. aureus.

\section{Antioxidant bioassay}

The ligand precursors P2HPA, P2NPA and their metal complexes (1-12) were tested for anti-oxidant activity by DPPH method. ${ }^{39}$ For antioxidant activity, the percentage inhibition of all the compounds are given in Table 2 and graphical representation in Figure 5. The results showed that triorganotin(IV) complexes (4-6, 10-12) demonstrated more activity with the inhibition values in the range of 90.13-87.24\% than the other diorganotin(IV) metal complexes, while it was observed that complexes showed much better values than the ligands. The effect of triorganotin(IV) aggregates on DPPH radical scavenging might be due to their hydrogen donation capacity. A possible exchanging mechanism was observed in such cases which can donate a proton and convert free radical DPPH to stable DPPH-H. The triorganotin(IV) complexes (4-6, 10-12) were most capable for radical exchange with DPPH thus resulting in enhanced antioxidant activity. The order of antioxidant inhibition can be concluded as triorganotin $>$ dimer $>$ monomer $>$ ligand.

\section{Antidiabetic bioassay}

In the present study 3-phthalimido-3(2-hydroxyphenyl) propanoic acid (P2HPA) and their organotin(IV) complexes (1-6) were evaluated for their in vivo antidiabetic activity in diabetic rabbits induced by alloxan. ${ }^{70}$ The study was
Table 2. Antioxidant bioassay

\begin{tabular}{lcc}
\hline Compound & $\begin{array}{c}\text { Concentration / } \\
\left(\mathrm{mg} \mathrm{mL}^{-1}\right)\end{array}$ & $\begin{array}{c}\text { Inhibition by DPPH } \\
\text { method }^{\mathrm{a}} \%\end{array}$ \\
\hline $\mathbf{1}$ & 0.5 & $89.61 \pm 0.61$ \\
$\mathbf{2}$ & 0.5 & $87.23 \pm 0.45$ \\
$\mathbf{3}$ & 0.5 & $85.25 \pm 0.31$ \\
$\mathbf{4}$ & 0.5 & $93.13 \pm 0.11$ \\
$\mathbf{5}$ & 0.5 & $91.51 \pm 0.46$ \\
$\mathbf{6}$ & 0.5 & $94.35 \pm 0.71$ \\
$\mathbf{7}$ & 0.5 & $88.28 \pm 0.43$ \\
$\mathbf{8}$ & 0.5 & $85.41 \pm 0.51$ \\
$\mathbf{9}$ & 0.5 & $87.47 \pm 0.32$ \\
$\mathbf{1 0}$ & 0.5 & $93.35 \pm 0.61$ \\
$\mathbf{1 1}$ & 0.5 & $92.15 \pm 0.11$ \\
$\mathbf{1 2}$ & 0.5 & $93.24 \pm 0.32$ \\
P2HPA & 0.5 & $70.31 \pm 1.62$ \\
P2NPA & 0.5 & $78.93 \pm 1.32$ \\
Standard & 0.1 & $97.34 \pm 0.33$ \\
\hline
\end{tabular}

${ }^{a}$ The percentage inhibition values represent the mean value of the three

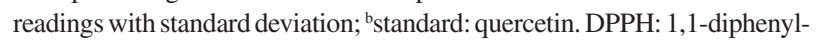
2-picrylhydrazyl radical; P2HPA: 3-phthalimido-3(2-hydroxyphenyl) propanoic acid; P2NPA: 3-phthalimido-3(2-nitrophenyl) propanoic acid.

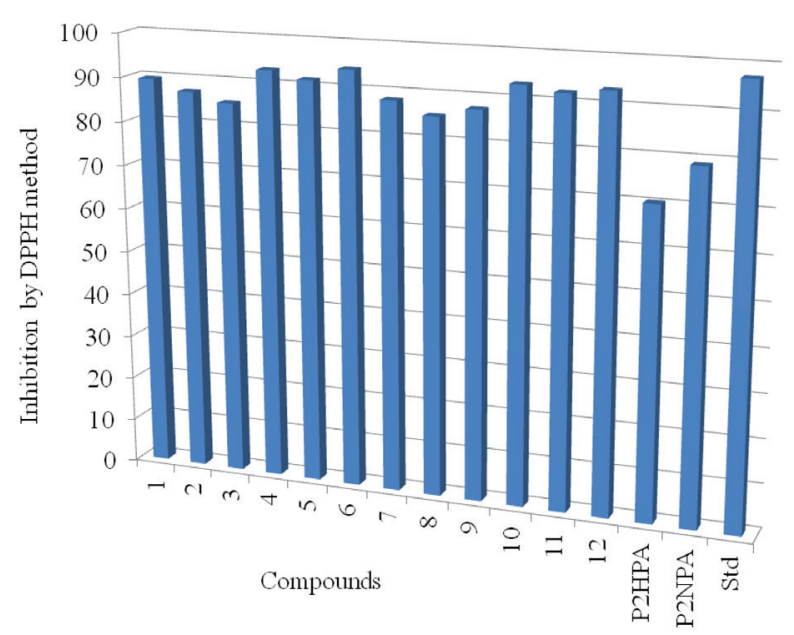

Figure 5. Comparison of percentage of inhibition values of compounds by DPPH method.

administered on eight groups (GI-GVIII) having 3 rabbits in each group to estimate the hypoglycemic effect of 3 increasing doses $\left(0.5,1.0\right.$ and $1.5 \mathrm{mg} \mathrm{kg}^{-1}$ of body weight). The results were collected and for reference the experimental groups (GI-GVII) results were compared with the control group (GVIII) having metformin injected rabbits. The grouping of animals and the results obtained are summarized in Table 3 and comparison of average blood glucose level of three increasing concentrations is given in Figure 6. 
Table 3. Antidiabetic bioassay

\begin{tabular}{|c|c|c|c|c|c|c|c|c|c|}
\hline \multirow{3}{*}{$\begin{array}{l}\text { No. of rabbit } \\
\text { groups }\end{array}$} & \multirow{3}{*}{$\begin{array}{c}\text { Compound } \\
\text { No. }\end{array}$} & \multicolumn{8}{|c|}{ Average blood glucose level / $\left(\mathrm{mg} \mathrm{dL}^{-1}\right)$} \\
\hline & & \multirow{2}{*}{$\begin{array}{l}\text { Before } \\
\text { alloxan }\end{array}$} & \multirow{2}{*}{$\begin{array}{l}\text { After alloxan } \\
\quad(24 \mathrm{~h})\end{array}$} & \multirow{2}{*}{$\begin{array}{c}\text { Concentration of } \\
\text { compound / } \\
\text { (mg kg } \\
\text { body weight })\end{array}$} & \multicolumn{5}{|c|}{ Results after treatment } \\
\hline & & & & & $3 \mathrm{~h}$ & $6 \mathrm{~h}$ & $9 \mathrm{~h}$ & $12 \mathrm{~h}$ & $24 \mathrm{~h}$ \\
\hline & & & & 0.5 & 227 & 199 & 110 & 118 & 211 \\
\hline \multirow[t]{3}{*}{ GI } & 1 & 78.2 & 311 & 1.0 & 1.0 & 190 & 122 & 104 & 170 \\
\hline & & & & 1.5 & 147 & 101 & 99 & 210 & 290 \\
\hline & & & & 0.5 & 210 & 169 & 105 & 140 & 254 \\
\hline \multirow[t]{3}{*}{ GII } & 2 & 88 & 305 & 1.0 & 198 & 142 & 115 & 190 & 290 \\
\hline & & & & 1.5 & 170 & 134 & 109 & 240 & 300 \\
\hline & & & & 0.5 & 290 & 209 & 155 & 240 & 264 \\
\hline \multirow[t]{3}{*}{ GIII } & 3 & 86 & 310 & 1.0 & 248 & 222 & 145 & 195 & 275 \\
\hline & & & & 1.5 & 189 & 144 & 110 & 240 & 290 \\
\hline & & & & 0.5 & 240 & 219 & 175 & 260 & 264 \\
\hline \multirow[t]{3}{*}{ GIV } & 4 & 96 & 320 & 1.0 & 278 & 220 & 145 & 245 & 275 \\
\hline & & & & 1.5 & 208 & 194 & 130 & 190 & 240 \\
\hline & & & & 0.5 & 250 & 229 & 165 & 240 & 264 \\
\hline \multirow[t]{3}{*}{ GV } & 5 & 106 & 303 & 1.0 & 248 & 212 & 165 & 195 & 245 \\
\hline & & & & 1.5 & 198 & 164 & 135 & 230 & 270 \\
\hline & & & & 0.5 & 265 & 230 & 165 & 240 & 294 \\
\hline \multirow[t]{3}{*}{ GVI } & 6 & 97 & 296 & 1.0 & 300 & 247 & 145 & 195 & 268 \\
\hline & & & & 1.5 & 295 & 244 & 154 & 205 & 242 \\
\hline & & & & 0.5 & 298 & 295 & 215 & 205 & 198 \\
\hline \multirow[t]{3}{*}{ GVII } & P2HPA & 75 & 293 & 1.0 & 332 & 310 & 245 & 215 & 175 \\
\hline & & & & 1.5 & 345 & 314 & 210 & 189 & 170 \\
\hline & & & & 0.5 & 265 & 235 & 104 & 190 & 250 \\
\hline \multirow[t]{2}{*}{ GVIII } & metformin & 89 & 305 & 1.0 & 245 & 225 & 135 & 165 & 215 \\
\hline & & & & 1.5 & 169 & 140 & 105 & 140 & 190 \\
\hline
\end{tabular}

All alloxan induced rabbits were randomly divided into eight groups $(\mathrm{n}=8)$; metformin was used as standard drug and served as a positive control. P2HPA: 3-phthalimido-3(2-hydroxyphenyl) propanoic acid.

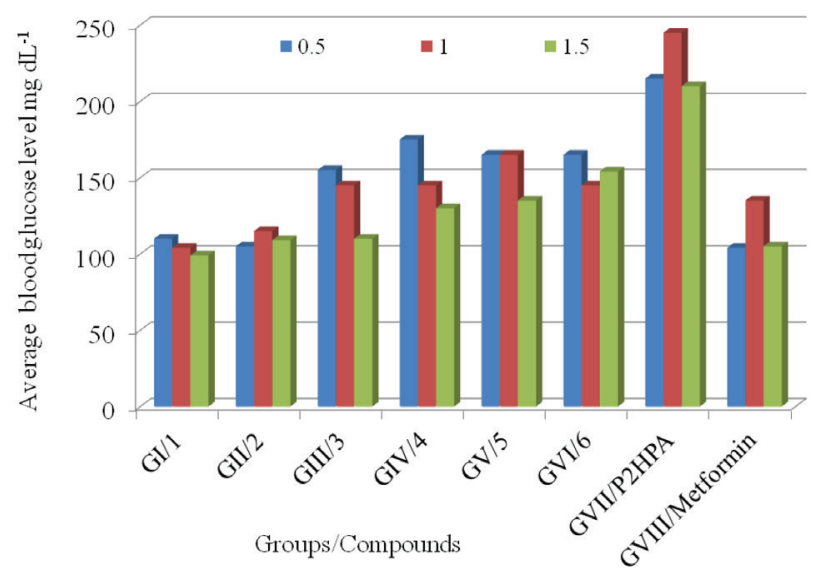

Figure 6. Comparison of the average blood glucose level of eight testing groups at three different concentrations after $9 \mathrm{~h}$ of time interval.

After 3, 6, 9, 12, and $24 \mathrm{~h}$ of treatment, a considerable trend in blood glucose level was noticed..$^{71}$ The blood sugar level dropped significantly by treatment with complexes (1-6) in (GI-GVI), respectively. This decrease was more significant with high dose of test solutions. The high dose effect of the complexes in GI-GVI was almost similar to metformin effect. The blood sugar level dropped down quickly at 3 and $6 \mathrm{~h}$ stabilized at the $9 \mathrm{~h}$ to about $175-105 \mathrm{mg} \mathrm{dL}^{-1}$ and then increased after $24 \mathrm{~h}$ of treatment. The possible reason for the increase of glucose level in the rabbit body was probably due to the decrease of injected compounds through metabolic excretion from the body. In case of ligand in GVII an opposite trend was seen. At first, the blood sugar levels increased rapidly, almost came to the normal levels with the passage of time and then stabilized between $198-170 \mathrm{mg} \mathrm{dL}^{-1}$ after $24 \mathrm{~h}$ of treatment. Thus, we can conclude that complexes 1-6 seemed more effective in lowering the blood sugar level as metformin in diabetic rabbits probably due to chelation or the addition of substrate. On the other hand, a noticeable variation was also observed among blood sugar level trends in all the experimental groups (GI-GVII) of diabetic rabbits. ${ }^{72}$

\section{Conclusions}

$N$-Phthalimido derivatives of $\beta$-amino acids and monomeric $(\mathbf{1}, \mathbf{3}, \mathbf{7}, \mathbf{9})$, dimeric $(\mathbf{2}, \mathbf{8})$ and triorganotin(IV) 
complexes (4-6, 10-12) were synthesized and characterized by elemental analysis, FTIR, ${ }^{1} \mathrm{H}$ NMR, ${ }^{13} \mathrm{C}$ NMR, ${ }^{119} \mathrm{Sn}$ NMR spectroscopy and mass spectrometry. The FTIR spectra of complexes confirmed the deprotonation of ligand precursors by the vanishing of $v(\mathrm{OH})$ peak at $3300-2500 \mathrm{~cm}^{-1}$. The ${ }^{1} \mathrm{H}$ NMR spectra of organotin(IV) complexes also confirmed their synthesis by deprotonation because the downfield region showed the absence of chemical shift for $-\mathrm{OH}$ proton at $12.00-10.00 \mathrm{ppm}$. The ${ }^{13} \mathrm{C}$ NMR, ${ }^{119} \mathrm{Sn}$ NMR and mass spectrometric data were also in agreement with FTIR and ${ }^{1} \mathrm{H}$ NMR illustrations. The results verified monodentate coordination of the triorganotin(IV) complexes with tetrahedral geometry in solid form and bidentate coordination with trigonal bipyramidal geometry in solution form. Diorganotin(IV) complexes exhibited bidentate coordination with transoctahedral geometry as monomers and a hexa-coordinated geometry as dimmers.

In general, ligands showed excellent predicted binding interactions which proved that such type of ligands can interact with the catalytic pocket of sortase A, (PDB ID $1 \mathrm{~T} 2 \mathrm{~W}$ ) an enzyme of $S$. aureus (MRSA), as evaluated by the docking studies. The biological screening of synthesized compounds revealed that metal complexes here presented were biologically more active than their isolated ligands and exhibited significant antibacterial, antioxidant and antidiabetic activities. Thus, we can conclude, on the broad perspective, that they can further be useful for humankind as antimicrobial, antioxidant and antihyperglycemic agents after their detailed and careful investigation.

\section{Supplementary Information}

Supplementary information (FTIR, ${ }^{1} \mathrm{H}$ NMR, ${ }^{13} \mathrm{C}$ NMR and EI-MS of the synthesized compounds) is available free of charge at http://jbcs.sbq.org.br as a PDF file.

\section{Acknowledgments}

The authors are very thankful to Institute of Chemical Sciences (Research Project No. DR \& EL/D-740 and research project No. DR \& EL/D-454), Bahauddin Zakariya University Multan Pakistan for providing research facilities. The Department of Chemistry, Quid-e-Azam University Islamabad Pakistan is also acknowledged for spectroscopic analysis.

\section{Author Contributions}

Muhammad M. Ahmed was responsible for conceptualization, data curation, formal analysis investigation, project administration, resources, software, validation, visualization, writing original draft, writing-review and editing; Nagina N. Riaz for data correct ration, formal analysis, investigation, software, visualization, writing original draft, writing-review and editing; Muhammad Kashif for conceptualization, data curation, investigation, software, visualization, writing-review and editing; Muhammad Ashfaq for formal analysis, investigation, project administration, software, validation, visualization, writing-review and editing; Muhammad Sajid for data curation, software, validation, visualization, writing-review and editing; Muhammad N. Arshad for data curation, formal analysis, investigation, visualization, writing original draft, writing-review and editing.

\section{References}

1. Ghazi, D.; Rasheed, Z.; Yousif, E.; Arch. Org. Inorg. Chem. Sci. 2018, 3, 344 .

2. Kemmer, M.; Dalil, H.; Biesemans, M.; Martins, J. C.; Mahieu, B.; Horn, E.; de Vos, D.; Tiekink, E. R. T.; Willem, R.; Gielen, M.; J. Organomet. Chem. 2000, 608, 63.

3. Alama, A.; Tasso, B.; Novelli, F.; Sapratore, F.; Drug Discovery Today 2009, 14, 500 .

4. Gielen, M.; Met.-Based Drugs 1995, 2, 99.

5. Sreeja, P. B.; Kurup, M. R. P.; Spectrochim. Acta, Part A 2005, $61,331$.

6. Singh, R.; Kaushik, N. K.; Spectrochim. Acta, Part A 2006, 65, 950.

7. Masudur Rehman, M.; Jusoh, I.; Abu Affan, M.; Sinin Hamdan, H.; Eur. J. Wood Wood Prod. 2013, 71, 463.

8. Tushar, S.; Baul, B.; Masharing, C.; Basu, S.; Rivarola, E.; Holcapek, M.; Jirasko, R.; Lycka, A.; de Vos, D.; Linden, A.; J. Organomet. Chem. 2006, 691, 952.

9. Patocka, J.; Mil. Med. Sci. Lett. 2011, 80, 2.

10. Kuhl, A.; Hahn, M. G.; Dumic, M.; Mittendorf, J.; Amino Acids 2005, 29, 89 .

11. Bonmatin, J. M.; Laprevote, O.; Peypoux, F.; Comb. Chem. High Throughput Screening 2003, 6, 541.

12. Cabrele, C.; Martinek, T. A.; Reiser, O.; Berlick, L.; J. Med. Chem. 2014, 57, 9718.

13. Fulop, F.; Martinek, T. A.; Toth, G. K.; Chem. Soc. Rev. 2006, $35,323$.

14. World Health Organization (WHO); List of Blueprint Priority Diseases; WHO: Cambridge, 2018, available at http://www. emro.who.int/pandemic-epidemic-diseases/news/list-ofblueprint-priority-diseases.html, accessed in December 2020.

15. Cosgrove, S. E.; Qi, Y.; Kaye, K. S.; Harbarth, S.; Karchmer, A. W.; Carmeli, Y.; Infect. Control Hosp. Epidemiol. 2005, 26, 166.

16. Ventola, C. L.; Pharm. Ther. 2015, 40, 277. 
17. Aslam, B.; Wang, W.; Arshad, M. I.; Khurshid, M.; Muzammil, S.; Rasool,M.H.;Salamat,M. K.F.; Infect.Drug Resist. 2018, 11, 1645.

18. Harman, D.; Ann. N. Y. Acad. Sci. 1998, 854, 1.

19. Maxwell, S. R. J.; Drugs 1995, 49, 345.

20. Sakurai, H.; Katoh, A.; Kiss, T.; Jakusch, T.; Hattori, M.; Metallomics 2010, 2, 670.

21. Jong-Anurakkun, N.; Bhandari, M. R.; Kawabata, J.; Food Chem. 2007, 103, 1319.

22. Rawat, V.; Singhai, M.; Kumar, A.; Jha, P. K.; Goyal, R.; North Am. J. Med. Sci. 2012, 4, 563.

23. Zavyalov, S. I.; Dorofeeva, O. V.; Rumyantseva, E. E.; Kolikova, L. B.; Ezhova, G. I.; Kravchenko, N. E.; Zavozin, A. G.; Pharm. Chem. J. 2002, 36, 440.

24. Spirig, T.; Weiner, E. M.; Clubb, R. T.; Mol. Microbiol. 2011, 82, 1044.

25. Furniss, B.; Hannaford, A. J.; Smith, P. W. G.; Tatchell, A. R.; Vogel's Text Book of Practical Organic Chemistry, $5^{\text {th }}$ ed.; Longman Scientific and Technical: New York, 1989, p. 510.

26. Juaristi, E.; Soloshonok, V. A.; Enantioselective Synthesis of Beta-Amino Acids; John Wiley \& Sons: Hoboken, New Jersey, USA, 2005.

27. Juaristi, E.; Enantioselective Synthesis of $\beta$-Amino Acids, $6^{\text {th }}$ ed.; Wiley-VCH: New York, 1997, p. 492.

28. Faghihi, K.; Feyzi, A.; Hajibeygi, M.; Des. Monomers Polym. 2012, 15, 523.

29. Faghihi, K.; Moghanian, H.; Chin. J. Polym. Sci. 2010, 28, 695.

30. Gielen, M.; Appl. Organomet. Chem. 2002, 16, 481.

31. Ashfaq, M.; Ahmed, M. M.; Shaheen, S.; Oku, H.; Mehmood, K.; Khan, A.; Niazi, S. B.; Ansari, T. M.; Jabbar, A.; Khan, M. I.; Inorg. Chem. Commun. 2011, 14, 5.

32. Ashfaq, M.; J. Organomet. Chem. 2006, 691, 1803.

33. Hans, K.; Pervez, M.; Ashfaq, M.; Anwar, S.; Badshah, A.; Majeed, A.; Acta Crystallogr., Sect. E: Crystallogr. Commun. 2002, 58, 466.

34. Aneja, K. R.; Sharma, C.; Joshi, R.; Jundishapur J. Microbiol. 2011, 4, 175.

35. Uddin, R.; Lodhi, M. U.; Ul-Haq, Z.; Chem. Biol. Drug Des. 2012, $80,300$.

36. Morris, G. M.; Huey, R.; Lindstrom, W.; Sanner, M. F.; Belew, R. K.; Goodsell, D. S.; Olson, A. J.; J. Comput. Chem. 2009, 16, 2785.

37. The Marvin Sketch, 17.28 software; ChemAxon, Budapest, Hungary, 2017; Trott, O. E.; Olson, A.; J. Comput. Chem. 2010, $31,455$.

38. Discovery Studio Modeling Environment, release 2017; BIOVIA, Dassault Systèmes, San Diego, 2019.

39. Williams, W. B.; Cuvelier, M. E.; Berset, C.; LWT-Food Sci. Technol. 1995, 28, 25.

40. Akthar, A. K.; Planta Med. 1981, 42, 205.

41. Ramanarayana Reddy, R. V.; Raja, T. A. R.; Uma Maheshwara Rao, K.; World J. Pharm. Res. 2014, 3, 4739.
42. Khan, M. I.; Baloch, M. K.; Ashfaq, M.; Gul, S.; J. Braz. Chem. Soc. 2009, 20, 341.

43. Costa, R. F. F.; Rebolledo, A. P.; Matencio, T.; Calado, H. D. R.; Ardisson, J. D.; Cortes, M. E.; Rodrigues, B. L.; Beraldo, H.; J. Coord. Chem. 2005, 58, 1307.

44. Khan, M. I.; Baloch, M. K.; Ashfaq, M.; J. Enzyme Inhib. Med. Chem. 2007, 22, 343.

45. Khan, M. I.; Baloch, M. K.; Ashfaq, M.; Rehmat, M.; Main Group Met. Chem. 2006, 29, 201.

46. Hussain, H.; Ahmad, V. U.; Green, I. R.; Krohn, K.; Hussain, J.; Badshah, A.; ARKIVOC 2007, 14, 289.

47. Camacho-Camacho, C.; Rojas-Oviedo, I.; Paz-Sandoval, M. A.; Cardenas, J.; Toscano, A.; Gielen, M.; Sosa, L. B.; Bartez, F. S.; Gracia-Mora, I.; Appl. Organomet. Chem. 2008, 22, 171.

48. Xueqing, S.; Christopher, C.; George, E.; Main Group Met. Chem. 2002, 25, 13.

49. Dalil, H.; Biesemans, M.; Teerenstra, M.; Willem, R.; Angiolini, L.; Salatelli, E.; Caretti, D.; Macromol. Chem. Phys. 2000, 201, 1266.

50. Ashfaq, M.; Majeed, A.; Rauf, A.; Khanzada, A. W. K.; Shah, W. U.; Ansari, M. I.; Bull. Chem. Soc. Jpn. 1999, 72, 2073.

51. Ahmad, A.; Khan, A.; Ali, S.; Parwez, M.; Acta Crystallogr., Sect. E: Crystallogr. Commun. 2006, 62, 1088.

52. Ashfaq, M.; Khan, M. I.; Baloch, M. K.; Malik, A.; J. Organomet. Chem. 2004, 689, 238.

53. Kemmer, M.; Ghys, L.; Gielen, M.; Biesemans, M.; Tiekink, E. R. T.; Willem, R.; J. Organomet. Chem. 1999, 582, 195.

54. Song, X.; Xie, Q.; Fang, X.; Heteroat. Chem. 2002, 13, 592.

55. Khan, M. I.; Baloch, M. K.; Ashfaq, M.; Appl. Organomet. Chem. 2006, 20, 463.

56. Kovala-Demertzi, D.; Dokorou, V. N.; Jasinski, J. P.; Opolski, A.; Wiecek, J.; Zervou, M.; Demertzis, M. A.; J. Organomet. Chem. 2005, 690, 1800.

57. Azadmeher, A.; Amini, M. M.; Hadipour, N.; Khavasi, H. R.; Fun, H. K.; Chen, C.-J; Appl. Organomet. Chem. 2008, 22, 19.

58. Pruchnik, F. P.; Banbula, M.; Ciunik, Z.; Latocha, M.; Skop, B.; Wilczok, T.; Inorg. Chim. Acta 2003, 356, 62.

59. Angiolini, L.; Caretti, D.; Mazzocchetti, L.; Salatelli, E.; Femoni, C.; J. Organomet. Chem. 2004, 689, 3301.

60. Gielen, M.; Bouhdid, A.; Willem, R.; Bregadze, V. I.; Ermanson, L. V.; Tiekink, E. R. T.; J. Organomet. Chem. 1995, 501, 277.

61. Singh, H. L.; Singh, J.; Bioinorg. Chem. Appl. 2014, 2014, ID 716578.

62. Gielen, M.; Joosen, E.; Mancilla, T.; Jurkschat, K.; Willem, R.; Roobol, C.; Bernheim, J.; Atassi, G.; Huber, F.; Hoffmann, E.; Preut, H.; Mahieu, B.; Main Group Met. Chem. 1995, 18, 27.

63. Shahzadi, S.; Shahid, K.; Ali, S.; Bakhtiar, M.; Turk. J. Chem. 2008, 32, 333.

64. Shahid, K.; Ali, S.; Shahzadi, S.; Badshah, A.; Khan, K. M.; Maharvi, G. M.; Synth. React. Inorg. Met.-Org. Chem. 2003, 33, 1221. 
65. Masood, M. T.; Ali, S.; Danish, M.; Mazhar, M.; Synth. React. Inorg. Met.-Org. Chem. 2002, 32, 9.

66. Bhatti, M. H.; Ali, S.; Masood, H.; Mazhar, M.; Qureshi, S. I.; Synth. React. Inorg. Met.-Org. Chem. 2000, 30, 1715.

67. Gielen, M.; Lelieveld, P.; de Vos, D.; Pan, H.; Willem, R.; Siesemans, M.; Fiebig, H. H.; Inorg. Chim. Acta 1992, 196, 115.

68. Li, J.; Zhao, G.; Xiong, C.; Ma, Y.; Synth. React. Inorg. Met.Org. Chem. 2001, 31, 85 .

69. Ali, S.; Ahmad, F.; Mazhar, M.; Munir, A.; Masood, M. T.; Synth. React. Inorg. Met.-Org. Chem. 2002, 32, 357.
70. Vangoori, Y.; Mishra, S. S.; Ambudas, B.; Ramesh, P.; Meghavani, G.; Deepika, K.; Prathibha, A.; Int. J. Med. Res. Health Sci. 2013, 2, 137.

71. Rajesh, P.; Sharon Sonia, S. S.; Reddy, Y. V.; Kumar, M. S.; Int. J. Pharm. Pharm. Sci. 2015, 2, 21.

72. Desai, J.; Somani, R.; Jain, K.; Indian J. Pharmacol. 2006, 66, 44.

Submitted: September 29, 2020

Published online: January 19, 2021 